\title{
Decomposition of nuclear magnetic resonance spin-spin coupling constants into active and passive orbital contributions
}

\author{
Jürgen Gräfenstein, Tell Tuttle, and Dieter Cremer ${ }^{a}$ ) \\ Department of Theoretical Chemistry, Göteborg University Reutersgatan 2, S-41320 Göteborg, Sweden
}

(Received 6 January 2004; accepted 2 March 2004)

\begin{abstract}
The theory of the $\mathbf{J}-\mathbf{O C}-\mathrm{PSP}$ (decomposition of $\mathrm{J}$ into orbital contributions using orbital currents and partial spin polarization) method is derived to distinguish between the role of active, passive, and frozen orbitals on the nuclear magnetic resonance (NMR) spin-spin coupling mechanism. Application of J-OC-PSP to the NMR spin-spin coupling constants of ethylene, which are calculated using coupled perturbed density functional theory in connection with the B3LYP hybrid functional and a $[7 s, 6 p, 2 d / 4 s, 2 p]$ basis set, reveal that the well-known $\pi$ mechanism for Fermi contact (FC) spin coupling is based on passive $\pi$ orbital contributions. The $\pi$ orbitals contribute to the spin polarization of the $\sigma$ orbitals at the coupling nuclei by mediating spin information between $\sigma$ orbitals (spin-transport mechanism) or by increasing the spin information of a $\sigma$ orbital by an echo effect. The calculated $\mathrm{FC}(\pi)$ value of the $\operatorname{SSCC}{ }^{1} \mathrm{~J}(\mathrm{CC})$ of ethylene is $4.5 \mathrm{~Hz}$ and by this clearly smaller than previously assumed. (C) 2004 American Institute of Physics.
\end{abstract}

[DOI: 10.1063/1.1711598]

\section{INTRODUCTION}

Nuclear magnetic resonance (NMR) parameters such as chemical shieldings or spin-spin coupling constants (SSCCs) provide important information about geometry, conformation, and other properties of a molecule. ${ }^{1-8}$ In general, magnetic parameters represent hypersensitive antennae that indicate features of the electronic structure which are difficult to detect by other means (e.g., degree of electron delocalization in weakly aromatic or antiaromatic molecules; anisotropy of the electron distribution, density close to the nucleus, polarizability of the electrons, detection of electron currents, etc.). The use of the magnetic properties of a molecule as suitable descriptors for electronic structure features requires of course that the dependence of a magnetic parameter on other molecular properties is known and can be described with simple mathematical relationships. Convincing examples in this regard are the Karplus relationship (dependence of the NMR spin-spin coupling constant $J$ on the conformation of a molecule), ${ }^{9}$ the dependence of $J$ on the $s$-character of a bond, ${ }^{10-13}$ charge-chemical shift relationships, ${ }^{14,15}$ etc. Although many of the frequently used relationships are made plausible on an ad hoc basis, a basic understanding in terms of spin density distributions, electron excitations, electron currents, and intramolecular magnetic fields is missing.

The present work is part of a larger project aimed at developing the theory for calculating SS-CCs with the help of DFT, ${ }^{16}$ using SSCCs as conformational descriptors, ${ }^{17-19}$ and analyzing the mechanism of NMR spin-spin coupling in dependence of the electronic structure of a molecule and its bonding characteristics. ${ }^{20-28}$ Indirect spin-spin coupling is transmitted by four different mechanisms from a nucleus B,

\footnotetext{
a) Author to whom correspondence should be addressed. Electronic mail: cremer@theoc.gu.se
}

which by its magnetic spin moment perturbs the surrounding electron density (perturbing nucleus), to the nucleus $\mathrm{A}$, the magnetic moment of which receives the perturbation of the electron density and responds to it (responding nucleus): ${ }^{29}$ (1) The Fermi contact (FC) mechanism caused by the internal magnetic field of nucleus $B$, which causes spin polarization of the density at the contact surface of this nucleus. Spin polarization travels like a wave through the electron system and interacts with the spin moment of nucleus A. (2) The spin dipole (SD) mechanism, which arises from the spin polarization caused by the external magnetic field of nucleus B. (3) The spin-orbit (SO) mechanisms are associated with orbital currents (equivalent to electron currents) generated by the spin moment of nucleus B; the electron currents are accompanied by a magnetic field, which is experienced by nucleus A. In the diamagnetic SO (DSO) case, there are circular currents corresponding to a Larmor precession for each electron, which depend on the form of the orbital and the associated orbital density in the molecular ground state. (4) In the paramagnetic SO (PSO) case, the orbital currents depend on the existence of appropriate excited states that can be reached from the ground state of the system by suitable excitations with not too large excitation energies.

Although the spin-spin coupling mechanism is independent of the direction in which the coupling information is moved from one nucleus to the other $(\mathrm{B} \rightarrow \mathrm{A}$ as used in this work or $\mathrm{A} \rightarrow \mathrm{B}$ ), the coupling mechanism can be more easily explained if one nucleus is always considered as the perturbing and a second nucleus is considered as the responding nucleus thus leading to the $\operatorname{SSCC}{ }^{n} \mathrm{~J}(\mathrm{AB})$ where $n$ gives the number of bonds in the shortest coupling path. If one considers organic molecules, for which most of the early NMR measurements were carried out, ${ }^{2-8}$ the four spin-spin coupling transmission mechanism associated with the Ramsey 
terms can be characterized according to the electrons (orbitals) involved in them.

The DSO mechanism involves all electrons. Although the individual components of the DSO term can be significant, they cancel each other largely out so that the DSO term in general is small and can be neglected. The sign and magnitude of the FC term depend on the properties of the $s \sigma$ spin density distribution at the responding nucleus ${ }^{20}$ whereas the sign and magnitude of the SD term are determined by the $p \sigma$ and $p \pi$-spin density distribution. ${ }^{22}$ The $p \sigma$ and $p \pi$ orbitals also determine the sign and magnitude of the PSO term although the transmission mechanism is now based on orbital currents generated by the perturbing nucleus and detected by the responding nucleus. ${ }^{21,24}$

At a first glance, it seems that $\pi$ electrons do not play any role for the FC spin coupling mechanism because these electrons have no spin density at the contact surface of a nucleus. Nevertheless, one knows that long range spin-spin coupling leading to sizable values of SSCCs ${ }^{4} J$ or ${ }^{5} J$ in conjugated hydrocarbons is dominated by a $\pi$ mechanism. ${ }^{4,5,30-36}$ Similar to the case of the ESR hyperfine coupling constants $a(\cdot \mathrm{C}-\mathrm{H})$ or $a(\cdot \mathrm{C}-\mathrm{C}-\mathrm{H})$, which result from spin polarization of the $\sigma$ system by an unpaired electron in a $\mathrm{C} p \pi$ orbital, ${ }^{37}$ the NMR spin-spin coupling mechanism is transferred via the $\pi$ electrons to the $s \sigma$ spin density at the responding nucleus. ${ }^{4,5,30-36}$

Clearly the $\pi$ electrons participate in the FC coupling mechanism in a passive rather than active way, i.e., they are not directly affected by the internal magnetic field of the perturbing nucleus. This passive contribution has been shown to be always present in any $\pi$ system where differing reports about the magnitude of the $\pi$ mechanism have been made. $^{4,5,30-36}$ In this work, we will focus on the $\pi$ mechanism, determine the magnitude of the $\mathrm{FC}(\pi)$ part of the total FC term, and describe the $\pi$ mechanism in detail. In this connection we will investigate the following two questions:

(1) In which ways can the $\pi$ orbitals contribute to the transmission of spin information between the coupling nuclei considering the fact that they can neither be polarized directly by the perturbing nucleus nor transfer spin information to the responding nucleus? How can the contribution of the $\pi$ orbitals to the FC coupling mechanism be quantified and how can it be visualized? For the purpose of answering these questions we will develop needed theory and computer software that can be routinely applied for any passively acting orbital.

(2) How important is the $\pi$ mechanism for SSCC across a double bond? Semiempirical calculations with a minimal basis set ${ }^{33-36}$ suggest that a significant portion of the FC part of ${ }^{1} \mathrm{~J}(\mathrm{CC}$ ) in unsaturated hydrocarbons (up to $20 \%$ or $15 \mathrm{~Hz}$ ) is related to contributions from the $\pi$ electrons. To what extent can these findings be confirmed by DFT calculations with an extended basis set? For the purpose of answering these questions, we will develop in Sec. II the theory of passive spin-spin coupling interactions and incorporate it into the recently described J-OC-PSP (decomposition of $J$ into orbital contributions using orbital currents and partial spin polarization) method. ${ }^{20}$ We will define active, passive, and frozen orbitals for the spin-spin coupling mechanism.
Furthermore, we will use the FC spin density distribution to visualize the $\pi$ mechanism. All calculations will employ the coupled perturbed density functional $(\mathrm{CP}-\mathrm{DFT})$ method $^{16}$ because we have shown that this is most suitable and reliable for larger hydrocarbons. ${ }^{15-19}$ In Sec. III, the $\pi$ mechanism will be discussed for the simple case of the SSCCs of ethylene. We will show the different modes of interaction between $\sigma$ and $\pi$ electrons and work out the general features for FC coupling via $\pi$ orbitals. These features will be verified by determining those calculational tools important for the description of the $\pi$ mechanism, which will provide a basis to compare previous calculations of the $\operatorname{FC}(\pi)$ part with the present one. We will show that the $\pi$ mechanism, although rather important for long-range coupling, does not play such an important role for short-range coupling as was previously assumed.

\section{THEORY OF THE SPIN-SPIN COUPLING MECHANISM}

CP-DFT used in this work to calculate NMR SSCC was described elsewhere. ${ }^{16}$ We will use CP-DFT in connection with the J-OC-PSP method ${ }^{20}$ to determine the orbital contributions to the four Ramsey terms. For this purpose, we briefly review the $\mathrm{CP}-\mathrm{DFT}$ equations, which for canonical orbitals read

$$
\left|\varphi_{k}^{(B)}\right\rangle=\sum_{a} \frac{\left\langle\varphi_{a}^{(0)}\left|F^{(B)}\right| \varphi_{k}^{(0)}\right\rangle}{\epsilon_{k}-\epsilon_{a}}\left|\varphi_{a}^{(0)}\right\rangle .
$$

Here, $F^{(B)}$ is the first-order KS operator for a perturbation $\mathrm{X}$ (X=PSO, FC, or SD) at nucleus $\mathrm{B}$, and $\left|\varphi_{k}^{(B)}\right\rangle$ are the firstorder orbitals corresponding to $F^{(B)}$. For brevity, we omit in this section the index $\mathrm{X}$ specifying the kind of perturbation. Furthermore, we will not distinguish explicitly between space and spin orbitals.

The analysis of SSCCs is done not in canonical MOs but in localized MOs (LMOs), which reflect the intuitive understanding of the electron system in the molecule (core orbitals, bonds, lone pairs). The LMOs $\psi_{k}$ are connected with the canonical MOs by an orthogonal transformation matrix $u$,

$$
\begin{aligned}
\left|\psi_{k}^{(0)}\right\rangle & =\sum_{l} u_{k l}\left|\varphi_{l}^{(0)}\right\rangle, \\
\left|\psi_{k}^{(B)}\right\rangle & =\sum_{l} u_{k l}\left|\varphi_{l}^{(B)}\right\rangle .
\end{aligned}
$$

Equations (2a) and (2b) are valid for not only LMOs but for any representation of the MOs. If LMOs are used the orbital energies $\epsilon_{k}$ must be replaced by the zeroth-order KS matrix $F_{k l}^{(0)}$, which leads to a coupling between the CP-DFT equations,

$$
\left|\psi_{k}^{(B)}\right\rangle=\sum_{a} \frac{\left\langle\psi_{a}^{(0)}\left|F^{(B)}\right| \psi_{k}^{(0)}\right\rangle-\sum_{l, l \neq k} F_{k l}^{(0)}\left\langle\psi_{a}^{(0)} \mid \psi_{l}^{(B)}\right\rangle}{F_{k k}^{(0)}-\epsilon_{a}}\left|\psi_{a}^{(0)}\right\rangle .
$$

For a derivation of Eq. (3), see Appendix A. The operator $F^{(B)}$ consists of the external perturbation $h^{(B)}$ and a contri- 
bution $\widetilde{F}^{(B)}$ that covers the feedback of the first-order orbitals to the $\mathrm{KS}$ operator. The latter can in turn be decomposed into contributions from the individual orbitals:

$$
\begin{aligned}
& F^{(B)}=h^{(B)}+\widetilde{F}^{(B)}, \\
& \widetilde{F}^{(B)}=\sum_{l} \widetilde{F}_{l}^{(B)}, \\
& \tilde{F}_{l}^{(B)}=\int \mathrm{d}^{3} r \frac{\delta F}{\delta \psi_{l}^{(0)}} \psi_{l}^{(B)}(\mathbf{r}) .
\end{aligned}
$$

With this decomposition, Eq. (3) takes the form

$$
\begin{aligned}
C_{a k}^{(B)}= & \frac{1}{F_{k k}^{(0)}-\epsilon_{a}}\left[h_{a k}^{(B)}+\left(\tilde{F}_{k}^{(B)}\right)_{a k}+\sum_{l, l \neq k}\left(\left(\tilde{F}_{l}^{(B)}\right)_{a k}\right.\right. \\
& \left.\left.-F_{k l}^{(0)} C_{a l}^{(B)}\right)\right] .
\end{aligned}
$$

For brevity, we have introduced the notation $C_{a k}^{(B)}$ $=\left\langle\psi_{a}^{(0)} \mid \psi_{k}^{(B)}\right\rangle$ and denote matrix elements of the form $\left\langle\psi_{p}^{(0)}|\hat{O}| \psi_{q}^{(0)}\right\rangle=O_{p q}$.

The matrix elements $\widetilde{F}_{a k}^{(B)}$ can be determined by comparing two equivalent expressions for the coefficient matrix $C_{a k}^{(B)}$. In general, it can be written as

$$
C_{a k}^{(B)}=-\sum_{k^{\prime} a^{\prime}}(\mathbf{H})_{a k, a^{\prime} k^{\prime}}^{-1} h_{a^{\prime} k^{\prime}}^{(B)} .
$$

Here, $\mathbf{H}$ is the full orbital Hessian, i.e., the matrix

$$
\mathbf{H}_{a k, a^{\prime} k^{\prime}}=\left.\frac{\partial^{2} E}{\partial C_{a k} \partial C_{a^{\prime} k^{\prime}}}\right|_{C=0}
$$

containing the second derivatives of the ground-state energy with respect to simultaneous orbital rotations $k \rightarrow a, k^{\prime}$ $\rightarrow a^{\prime}$. The explicit form of $\mathbf{H}$ can be found elsewhere. ${ }^{38}$ Equation (5) can be rearranged into

$$
C_{a k}^{(B)}=-\sum_{k^{\prime} a^{\prime}}\left[\mathbf{H}^{(0)}\right]_{a k, a^{\prime} k^{\prime}}^{-1} F_{a^{\prime} k^{\prime}}^{(B)},
$$

where

$$
\mathbf{H}_{a k, a^{\prime} k^{\prime}}^{(0)}=\sum_{k^{\prime \prime} a^{\prime \prime}} u_{k k^{\prime \prime}} u_{k^{\prime} k^{\prime \prime}} u_{a a^{\prime \prime}} u_{a^{\prime} a^{\prime \prime}}\left(\boldsymbol{\epsilon}_{a^{\prime \prime}}-\epsilon_{k^{\prime \prime}}\right)
$$

is the zeroth-order Hessian, which results from the full Hessian $\mathbf{H}$ by omitting all terms related to two-electron interactions. Note that $u_{a a^{\prime}}=\delta_{a a^{\prime}}$ and $\mathbf{H}^{(0)}$ will be diagonal in $a a^{\prime}$ if the virtual orbitals are kept canonical. In this special case, the zeroth-order Hessian takes the form

$$
\mathbf{H}_{a k, a^{\prime} k^{\prime}}^{(0)}=\delta_{a a^{\prime}}\left(\boldsymbol{\epsilon}_{a}-F_{k k^{\prime}}^{(0)}\right) .
$$

Comparing Eqs. (6) and (8) gives

$$
\begin{aligned}
& F_{a k}^{(B)}=\sum_{k^{\prime} a^{\prime}} \sum_{k^{\prime \prime} a^{\prime \prime}} \mathbf{H}_{a k, a^{\prime \prime} k^{\prime \prime}}^{(0)}(\mathbf{H})_{a^{\prime \prime} k^{\prime \prime}, a^{\prime} k^{\prime}}^{-1} h_{a^{\prime} k^{\prime}}^{(B)}, \\
& \widetilde{F}_{a k}^{(B)}=-\sum_{k^{\prime} a^{\prime}} \sum_{k^{\prime \prime} a^{\prime \prime}} \widetilde{\mathbf{H}}_{a k, a^{\prime \prime} k^{\prime \prime}}(\mathbf{H})_{a^{\prime \prime} k^{\prime \prime}, a^{\prime} k^{\prime}}^{-1} h_{a^{\prime} k^{\prime}}^{(B)} .
\end{aligned}
$$

Note that $\mathbf{H}$, and thus $\widetilde{\mathbf{H}}$, are not known explicitly in practical calculations. Rather, one rewrites Eq. (11b) with the help of Eq. (5) as

$$
\widetilde{F}_{a k}^{(B)}=-\sum_{k^{\prime} a^{\prime}} \tilde{\mathbf{H}}_{a k, a^{\prime} k^{\prime}} C_{a^{\prime} k^{\prime}}^{(B)} .
$$

Equations (5) and (12) represent a set of self-consistent equations for the $C_{a k}^{(B)}$ and the $\left(\widetilde{F}_{l}^{(B)}\right)_{a k}$. In the iterative solution of Eqs. (5) and (12), only a small part of $\widetilde{\mathbf{H}}$ is actually calculated.

Once the $C_{a k}^{(B)}$ have been determined, the SSCC can be calculated as

$$
\begin{aligned}
K_{\mathrm{AB}} & =\sum_{k} K_{\mathrm{AB}}^{k}, \\
K_{\mathrm{AB}}^{k} & =\left\langle\psi_{k}^{(B)}\left|h^{(A)}\right| \psi_{k}^{(0)}\right\rangle \\
& =\sum_{a} C_{a k}^{(B)} h_{a k}^{(A)} .
\end{aligned}
$$

Equations (13a) and (13b) provide a simple orbital decomposition of $K_{\mathrm{AB}}$, which will be discussed in more detail later in this section.

Equation (5) shows explicitly the different couplings between the orbitals. The first two terms in the square brackets on the right-hand side (rhs) of Eq. (5) are one-orbital terms. The first term describes the direct impact of the external perturbation $h^{(B)}$ on $\left|\psi_{k}\right\rangle$, whereas the second term reflects the feedback of $\left|\psi_{k}^{(B)}\right\rangle$ on itself via $\widetilde{F}_{k}^{(B)}$. Generally, both the Coulomb, the exchange, and the correlation part of $\widetilde{F}_{k}^{(B)}$ will be involved in this interaction. The correlation term is usually small compared to the exchange term. None of the magnetic perturbations accompanying spin-spin coupling change the total density, hence the Coulomb part of $\widetilde{F}_{k}$ vanishes, and the exchange term is dominating. Therefore we call this process self-exchange repulsion.

The third term in the square brackets of Eq. (5) comprises the two-orbital terms, which describes their mutual interactions invoked by the perturbation. The two terms are of a basically different nature.

(a) The first term, containing $\widetilde{F}_{l}^{(B)}$, describes how changes in orbital $\left|\psi_{l}\right\rangle$ via the KS operator influence $\left|\psi_{k}\right\rangle$. Similarly as for the self-exchange repulsion, this coupling is dominated by exchange effects whereas Coulomb effects are excluded and correlation effects play only a minor role. The interaction between the electrons in orbitals $k$ and $l$ is related to the steric repulsion known from classical chemistry: If orbital $l$ is modified, this modifies the conditions for favorable exchange interactions between $k$ and $l$, and $k$ responds with a change so as to reoptimize its equal-spin overlap and thus its exchange energy with $l$. The steric interactions between $k$ and $l$ are always present, no matter whether canonical or localized MOs are used.

(b) The second term, containing the nondiagonal elements of $F^{(0)}$, is not related directly to the perturbation. It does not describe any dynamic electron-electron interactions but a mutual influence of the orbitals that arises 
at the one-electron level due to the Pauli principle. It may be regarded as a resonance interaction between orbitals $k$ and $l$ and can be rationalized from the KohnSham equation in localized orbitals, which has the form

$$
\hat{F}\left|\psi_{k}\right\rangle=\sum_{l} F_{k l}\left|\psi_{l}\right\rangle
$$

Equation (14) shows that the Fock operator delocalizes the electron in orbital $k$ into all other occupied LMOs $l$. Due to the Pauli principle this delocalization is prohibited. However, if for $l \neq k$ one of the orbitals is perturbed either directly by the perturbing nucleus or indirectly through other orbitals, this delocalization will take effect, and the form of orbital $k$ will be affected as well. Only for a diagonal matrix $F_{k k}$, which corresponds to canonical orbitals, are the changes in the occupied orbitals independent of each other. The resonance terms reflect the fact that a local perturbation (as the nuclear spin at the perturbing nucleus) will affect the electron system in the whole molecule rather than just locally around the perturbation. Canonical (delocalized) orbitals can account for this effect directly whereas for localized orbitals the resonance terms are necessary to transport this effect through the electron system of the molecule. The resonance interaction may be important, e.g., for the transfer of the perturbation between neighboring $\sigma$ bond LMOs. For the transfer between $\sigma$ and $\pi$ orbitals it vanishes for reasons of symmetry.

The fact that Eq. (5) contains terms involving up to two orbitals corresponds to the fact that steric exchange and resonance interaction occur between pairs of electrons. Equation (5) allows in principle to decompose $C_{a k}^{(B)}$ into contributions related to orbital $k$ only and terms related to orbitals $k$ and $l$,

$$
\begin{aligned}
& \left(C_{k}^{(B)}\right)_{a k}=\frac{1}{F_{k k}^{(0)}-\epsilon_{a}}\left[h_{a k}^{(B)}+\left(\tilde{F}_{k}^{(B)}\right)_{a k}\right], \\
& \left(C_{k \leftarrow l}^{(B)}\right)_{a k}=\frac{1}{F_{k k}^{(0)}-\epsilon_{a}}\left[\left(\widetilde{F}_{l}^{(B)}\right)_{a k}-F_{k l}^{(0)} C_{a l}^{(B)}\right], \\
& K_{\mathrm{AB}}^{(k)}=\sum_{a}\left(C_{k}^{(B)}\right)_{a k} h_{a k}^{(A)}, \\
& K_{\mathrm{AB}}^{(k \leftarrow l)}=\sum_{a}\left(C_{k \leftarrow l}^{(B)}\right)_{a k} h_{a k}^{(A)}, \\
& K_{\mathrm{AB}}^{(k \leftrightarrow l)}=K_{\mathrm{AB}}^{(k \leftarrow l)}+K_{\mathrm{AB}}^{(l \leftarrow k)} .
\end{aligned}
$$

The notation $(k \leftarrow l)$ indicates that interactions are considered where the electron in orbital $k$ is influenced by the electron in orbital $l$. The mutual interaction term $(k \leftrightarrow l)$ is given by Eq. (15e).

A process that transfers spin information from the perturbing to the responding nucleus may comprise a chain of steric-exchange and/or resonance interactions and thus involve not only one or two orbitals but an arbitrary number of orbitals, which may occur one or several times in the chain. Examples for such chains, which will be called orbital paths henceforth, are

$$
(\mathrm{B}) \rightarrow k \rightarrow(\mathrm{A}),
$$

$$
\begin{aligned}
& (\mathrm{B}) \rightarrow k \rightarrow l \rightarrow(\mathrm{A}), \\
& (\mathrm{B}) \rightarrow k \rightarrow l \rightarrow m \rightarrow(\mathrm{A}), \\
& (\mathrm{B}) \rightarrow k \rightarrow l \rightarrow k \rightarrow(\mathrm{A}), \\
& (\mathrm{B}) \rightarrow k \rightarrow l \rightarrow l \rightarrow(\mathrm{A}), \\
& (\mathrm{B}) \rightarrow k \rightarrow l \rightarrow l \rightarrow k \rightarrow(\mathrm{A}), \\
& (\mathrm{B}) \rightarrow k \rightarrow l \rightarrow l \rightarrow k \rightarrow k \rightarrow(\mathrm{A}) .
\end{aligned}
$$

The contributions to $K_{\mathrm{AB}}$ from orbital paths containing more than two orbitals are included implicitly into the one- or two-orbital terms of Eq. (15). Equations (15c) and (15d) indicate that orbital $k$ is the one that interacts directly with the responding nucleus $\mathrm{A}$. Hence, $K_{\mathrm{AB}}^{(k)}$ and $K_{\mathrm{AB}}^{(k \leftarrow l)}$ together contain the contributions from all orbital paths where $k$ is the last orbital. Of these contributions, $K_{\mathrm{AB}}^{(k \leftarrow l)}$ contains those where $l$ is the second to last orbital as can be seen from Eq. (15b). $K_{\mathrm{AB}}^{(k)}$ summarizes the orbital path containing only $k$ and those orbital paths where $k$ is both the last and the second to last orbital in the orbital path. Orbital paths (16a) and (16g) are contained in $K_{\mathrm{AB}}^{(k)}$, (16e) in $K_{\mathrm{AB}}^{(l)}$, (16b) in $K_{\mathrm{AB}}^{(l \leftarrow k)}$, (16c) in $K_{\mathrm{AB}}^{(m \leftarrow l)}$, and (16f) in $K_{\mathrm{AB}}^{(k \leftarrow l)}$.

If the spin-spin coupling is dominated by one- and twoorbital paths as is the case for one- bond SSCCs in small molecules, ${ }^{20}$ then Eqs. (15) will provide the basis for an efficient orbital decomposition of $K_{\mathrm{AB}}$ on the basis of a conventional SSCC calculation. However, if coupling paths made up of three or more orbitals are of interest for the SSCC, this decomposition will have two disadvantages:

(1) It does not allow to separate the three-,..., n-orbital contributions from the one- and two- orbital contributions.

(2) It is asymmetric with respect to perturbing and responding nucleus.

It should be noted that (2) is also true for the orbital contributions introduced in Eqs. (13): $K_{\mathrm{AB}}^{k}$ contains all orbital paths that end with orbital $k$, i.e., the orbital decomposition is done exclusively with respect to the responding nucleus. Contributions $K_{\mathrm{AB}}^{k}$ will lead to a simple decomposition of SSCC $K_{\mathrm{AB}}$. The natural $J$-coupling (NJC) approach suggested by Peralta, Contreras, and Snyder ${ }^{39}$ (henceforth called NJC-1) decomposes the FC term in the spirit of Eqs. (13) and therefore requires a second calculation to symmetrize results. This is also true for an extension of NJC-1 suggested by Weinhold and co-workers ${ }^{40}$ and denoted here as NJC-2.

An orbital analysis of the SSCCs that avoids shortcomings (1) and (2) can be accomplished with the concept of passive and frozen orbitals discussed in the following section.

\section{A. Differentiation of active, passive, and frozen orbitals in the spin-spin coupling mechanism}

An orbital can participate in the transfer of spin information from the perturbing to the responding nucleus in three ways:

(1) The orbital can be modified directly by $h^{(B)}$. 
(2) The change in the orbital may be sensed by $h^{(A)}$ and thus contribute to $K_{\mathrm{AB}}$ directly.

(3) The orbital may be modified by other orbitals, and in turn modify other orbitals, via steric exchange or resonance interactions.

Orbitals for which (1) or (2) or both apply are called active orbitals, whereas those for which only (3) applies are called passive orbitals. Analogously, a contribution to the SSCCs that is related to (1) and (2) is called an active orbital contribution and one that is related to (3), a passive orbital contribution. An active orbital generally provides both an active and a passive contribution to the SSCC. Each orbital path contains one or two active orbitals at its two ends, and any passive contribution of some orbital is at the same time an active contribution of this or these two active orbitals.

Obviously, the first and last orbital in a coupling path are related to active contributions, all other orbitals in the path to passive contributions. Furthermore, only orbital paths that both start and end with an active orbital make nonzero contributions to the SSCC.

The J-OC-PSP1 analysis: The distinction between active and passive orbital contributions allows for a simple decomposition of $K_{\mathrm{AB}}$ into one- and two-orbital contributions in the following way: The one-orbital contribution for orbital $k$ comprises all orbital paths where only orbital $k$ is involved as an active orbital, i.e.,

$$
\begin{aligned}
& (\mathrm{B}) \rightarrow k \rightarrow(\mathrm{A}), \\
& (\mathrm{B}) \rightarrow k \rightarrow\{\cdots\} \rightarrow k \rightarrow(\mathrm{A}) .
\end{aligned}
$$

The symbol $\{\cdots\}$ stands for any number of intermediate steps (including the case of none at all). The two-orbital contribution for a pair of orbitals $(k, l)$ comprises all orbital paths where both $k$ and $l$ make active contributions, i.e., all paths of the form

$$
\begin{aligned}
& (\mathrm{B}) \rightarrow k \rightarrow\{\cdots\} \rightarrow l \rightarrow(\mathrm{A}), \\
& (\mathrm{B}) \rightarrow l \rightarrow\{\cdots\} \rightarrow k \rightarrow(\mathrm{A}) .
\end{aligned}
$$

The decomposition according to Eqs. (17) and (18) is realized in the simplest form of the J-OC-PSP approach, ${ }^{20}$ henceforth called J-OC-PSP1 to distinguish it from the more detailed orbital decomposition J-OC-PSP2 discussed in the following.

If orbital paths containing three or more steps are neglected, the one- and two-orbital terms according to Eqs. (17) and (18) will become equivalent to those given by Eqs. (15c) and (15e).

The J-OC-PSP1 orbital contributions can be determined on the basis of SSCC calculations that are modified in the way that the active contributions are retained only for selected orbitals, whereas the other orbitals are artificially set passive, i.e., their active contribution is eliminated. Setting orbital $k$ passive implies that both the matrix elements $h_{a k}^{(B)}$ and $h_{a k}^{(A)}$ (for all $a$ ) are set to zero. The CP-DFT equations take then the following form:

$$
\begin{aligned}
& C_{a k}^{(B), \mathrm{pass}}= \frac{1}{F_{k k}^{(0)}-\epsilon_{a}}\left[\left(h_{a k}^{(B), \mathrm{pass}}+\left(\widetilde{F}_{k}^{(B), \mathrm{pass}}\right)_{a k}\right)\right. \\
&\left.+\sum_{l, l \neq k}\left(\left(\widetilde{F}_{l}^{(B), \mathrm{pass}}\right)_{a k}-F_{k l}^{(0)} C_{a l}^{(B), \mathrm{pass}}\right)\right], \\
& h_{a k}^{(N), \mathrm{pass}}= \sum_{a^{\prime} k^{\prime}}\left(\delta_{a a^{\prime}} \delta_{k k^{\prime}}-P_{a k, a^{\prime} k^{\prime}}^{\mathrm{pass}}\right) h_{a^{\prime} k^{\prime}}^{(N)} \quad(N=A, B), \\
& \tilde{F}_{a k}^{(B), \mathrm{pass}}=-\sum_{k^{\prime} a^{\prime}} \widetilde{\mathbf{H}}_{a k, a^{\prime} k^{\prime}} C_{a^{\prime} k^{\prime}}^{(B), \mathrm{pass}}, \\
& K_{\mathrm{AB}}^{\mathrm{pass}}=\sum_{k a} C_{a k}^{(B), \mathrm{pass}} h_{a k}^{(A), \mathrm{pass}} .
\end{aligned}
$$

Here, $P^{\text {pass }}$ is a projection operator that eliminates all orbitals that are to become passive

$$
P_{a k, a^{\prime} k^{\prime}}^{\text {pass }}= \begin{cases}1 & \text { for } a=a^{\prime}, k=k^{\prime}, k \text { passive, } \\ 0 & \text { otherwise. }\end{cases}
$$

In the following, we will use the notation $K_{\mathrm{AB}}^{\mathrm{pass}}\left[k, k^{\prime}, \ldots\right]$ for the SSCC resulting from a calculation with orbitals $k, k^{\prime}, \ldots$ set passive, correspondingly we will use the notations $h^{(N) \text {,pass }}\left[k, k^{\prime}, \ldots\right](N=A, B) \quad$ and $\quad C^{(N) \text {, pass }}\left[k, k^{\prime}, \ldots\right](N$ $=A, B)$. Furthermore, we will employ the symbol $[\neg k]$ for a calculation in which only orbital $k$ is kept active whereas all other orbitals are set passive, and the symbol $[\neg(k, l)]$ for a calculation in which $k, l$ are kept active and all other orbitals set passive. In J-OC-PSP1, the one-orbital term is given by

$$
K_{\mathrm{AB}}^{\mathrm{PSP} 1}(k)=K_{\mathrm{AB}}^{\mathrm{pass}}[\neg k],
$$

where the superscript PSP1 is used as a shorthand notation for J-OC-PSP1. For the calculation of the two-orbital term $K_{\mathrm{AB}}^{\mathrm{PSP} 1}(k \leftrightarrow l)$, it has to be considered that $K_{\mathrm{AB}}^{\mathrm{pass}}[\neg(k, l)]$ contains all orbital paths that start and end with either orbital $k$ or orbital $l$, i.e., all orbital paths in Eqs. (17) and (18). $K_{\mathrm{AB}}^{\mathrm{PSP} 1}(k \leftrightarrow l)$ can therefore be calculated as the difference

$$
K_{\mathrm{AB}}^{\mathrm{PSP} 1}(k \leftrightarrow l)=K_{\mathrm{AB}}^{\mathrm{pass}}[\neg(k, l)]-K_{\mathrm{AB}}^{\mathrm{pass}}[\neg k]-K_{\mathrm{AB}}^{\mathrm{pass}}[\neg l] .
$$

In practice, the $K_{\mathrm{AB}}^{\mathrm{PS} 1}(k \leftrightarrow l)$ can be determined as a byproduct of the calculation of the one-orbital terms $K_{\mathrm{AB}}^{\mathrm{PSP} 1}(k)$. This reduces the number of orbital-selected SSCC calculations required for a J-OC-PSP1 analysis considerably. Details are found in Appendix B.

One can perform the J-OC-PSP1 analysis for groups of orbitals rather than individual orbitals, e.g., by decomposing the orbitals of a molecule in core orbitals, $\sigma$ bond orbitals along the coupling path, lone-pair orbitals at the coupling nuclei, other $\sigma$ bond orbitals not included into the coupling path, and $\pi$ orbitals. $^{20}$ In this way, the analysis is largely simplified whereas it still provides an efficient description of the coupling mechanism.

The J-OC-PSP2 analysis: A more advanced stage of the analysis, henceforth called J-OC-PSP2, has to be applied if one is interested in the contributions of passive orbitals, e.g., the $\pi$ contributions to the FC term in alkenes, polyenes, or aromatic molecules. Passive orbitals occur only 
in coupling paths comprising of at least three orbitals, and their contributions are distributed over those one- and twoorbital terms in J-OC-PSP1 that contain active orbitals only. The one- and two-orbital terms containing the passive orbitals are zero, hence J-OC-PSP1 gives the impression that the passive orbitals make no contribution to the SSCC. However, it has been known for a long time $e^{4,5,30-36}$ that, e.g., the $\pi$ orbitals play an important role for the FC spin-spin coupling mechanism in molecules with multiple bonds. For an explicit analysis of passive orbital contributions, one needs an orbital decomposition that explicitly contains three-, four-, etc., orbital terms, which is realized at the J-OC-PSP2 level.

In J-OC-PSP2, orbital paths will be classified not only based on the active contributions but also based on the passive ones. That is, the one-orbital contribution $K_{\mathrm{AB}}^{\mathrm{PSP}}(k)$ (PSP2 is a shorthand notation for J-OC-PSP2) contains all orbital paths where orbital $k$ is involved in any position, $K_{\mathrm{AB}}^{\mathrm{PSP} 2}(k \leftrightarrow l)$, all paths where both $k$ and $l$ are involved, $K_{\mathrm{AB}}^{\mathrm{PSP} 2}(k \leftrightarrow l \leftrightarrow m)$, all paths where all three orbitals $k, l, m$ are involved, etc.

Setting an orbital passive eliminates only its active contribution to the SSCC but leaves the passive ones intact. For the calculation of the J-OC-PSP2 orbital contributions, all contributions of a given orbital must be eliminated. This can be done by freezing the orbital, i.e., excluding all excitations from this orbital into the virtual space (equivalent to excluding it from the $\mathrm{CP}-\mathrm{DFT}$ calculation). Accordingly, the $\mathrm{CP}-$ DFT equations take the following form:

$$
\begin{aligned}
& C_{a k}^{(B), \text { froz }}= \frac{1}{F_{k k}^{(0)}-\epsilon_{a}}\left[\left(h_{a k}^{(B), \text { froz }}+\left(\widetilde{F}_{k}^{(B), \text { froz }}\right)_{a k}\right)\right. \\
&+\sum_{l, l \neq k}\left(\left(\widetilde{F}_{l}^{(B), \text { froz }}\right)_{a k}-\sum_{k^{\prime} a^{\prime}}\left(\delta_{a a^{\prime}} \delta_{k k^{\prime}}-P_{a k, a^{\prime} k^{\prime}}^{\mathrm{froz}}\right)\right. \\
&\left.\left.\times F_{k^{\prime} l}^{(0), \text { froz }} C_{a^{\prime} l}^{(B), \text { froz }}\right)\right] \\
& h_{a k}^{(N), \text { froz }=} \sum_{a^{\prime} k^{\prime}}\left(\delta_{a a^{\prime}} \delta_{k k^{\prime}}-P_{a k, a^{\prime} k^{\prime}}^{\mathrm{frrz}}\right) h_{a^{\prime} k^{\prime}}^{(N)} \quad(N=A, B), \\
& \widetilde{F}_{a k}^{(B), \text { froz }=}-\sum_{k^{\prime} a^{\prime}} \sum_{k^{\prime \prime} a^{\prime \prime}}\left(\delta_{a a^{\prime \prime}} \delta_{k k^{\prime \prime}}-P_{a k, a^{\prime \prime} k^{\prime \prime}}^{\mathrm{froz}}\right) \\
& \times \widetilde{\mathbf{H}}_{a^{\prime \prime} k^{\prime \prime}, a^{\prime} k^{\prime}} C_{a^{\prime} k^{\prime}}^{(B), \text { froz }}, \\
& K_{\mathrm{AB}}^{\mathrm{froz}=} \sum_{k a} C_{a k}^{(B), \text { froz } h_{a k}^{(A), \text { froz }} .}
\end{aligned}
$$

Here, $P^{\text {froz }}$ is defined in analogy to $P^{\text {pass }}$ of Eq. (20), i.e.,

$$
P_{a k, a^{\prime} k^{\prime}}^{\mathrm{froz}}= \begin{cases}1 & \text { for } a=a^{\prime}, k=k^{\prime}, k \text { frozen, } \\ 0 & \text { otherwise. }\end{cases}
$$

In distinction to the case of passive orbitals, the matrix elements in question are set to zero not only in $h^{(B) \text {, froz }}$ but also in $\widetilde{F}^{(B)}$,froz.

The calculation of the J-OC-PSP2 orbital contributions differs slightly from that of the J-OC-PSP1 terms. For in- stance, $K_{\mathrm{AB}}^{\mathrm{froz}}[\neg k]$ contains only the orbital paths $k, k \rightarrow k, k$ $\rightarrow k \rightarrow k$, etc., and is thus not equal to $K_{\mathrm{AB}}^{\mathrm{PS} 2}(k)$. Whereas the full $K_{\mathrm{AB}}$ comprises all orbital paths, $K_{\mathrm{AB}}^{\mathrm{froz}}[k]$ contains all orbital paths except those involving $k$ in any position. Therefore,

$$
K_{\mathrm{AB}}^{\mathrm{PS} 2}(k)=K_{\mathrm{AB}}-K_{\mathrm{AB}}^{\mathrm{froz}}[k] .
$$

The SSCC $K_{\mathrm{AB}}^{\mathrm{froz}}[k, l]$ contains all paths except those containing $k$ or $l$ (or both) in any position. Thus,

$$
\begin{aligned}
K_{\mathrm{AB}}^{\mathrm{PS} 2}(k \leftrightarrow l)=K_{\mathrm{AB}} & -K_{\mathrm{AB}}^{\mathrm{froz}}[k]-K_{\mathrm{AB}}^{\mathrm{froz}}[l]+K_{\mathrm{AB}}^{\mathrm{froz}}[k, l] \\
K_{\mathrm{AB}}^{\mathrm{PSP} 2}(k \leftrightarrow l \leftrightarrow m)= & K_{\mathrm{AB}}-K_{\mathrm{AB}}^{\mathrm{froz}}[k]-K_{\mathrm{AB}}^{\mathrm{froz}}[l]-K_{\mathrm{AB}}^{\mathrm{froz}}[m] \\
& +K_{\mathrm{AB}}^{\mathrm{froz}}[k, l]+K_{\mathrm{AB}}^{\mathrm{froz}}[k, m] \\
& +K_{\mathrm{AB}}^{\mathrm{froz}}[l, m]-K_{\mathrm{AB}}^{\mathrm{froz}}[k, l, m] .
\end{aligned}
$$

More complicated expressions result for four-orbital and higher orbital interaction terms, which in principal can all be calculated with J-OC-PSP2 although the information gained by these terms is small.

It should be noted that the J-OC-PSP orbital decomposition of the SSCC is done with respect to sets of unique orbitals occurring in an orbital path rather than with respect to individual orbital paths. For instance, the contributions from Eqs. (16b)- $(16 \mathrm{~g})$ will all be collected in $K_{\mathrm{AB}}^{\mathrm{PSP} 2}(k \leftrightarrow l)$. This is in line with the way orbital contributions to SSCCs are understood. If one were interested in the contributions to individual orbital paths one had to follow the iterative solution of the CP-DFT equations (without convergence acceleration) step by step and to modify the projector $P^{\text {froz }}$ in each step.

J-OC-PSP2 can lead to many orbital contributions, which imply additional calculations. The expenses of the J-OC-PSP2 analysis can, however, be reduced in two ways. First, one is often interested in just a few rather than all three- or more-orbital contributions because most of the three- or more-orbital contributions can be predicted to be negligible. Hence, one can restrict the J-OC-PSP2 to a few selected orbital combinations.

Second, one may be interested in the effect of some orbitals as a group rather than the contributions of the individual orbitals within the group. An example is the $\pi$ system in an alkene. In this case, one can determine the total contribution for a group of orbitals (all $\sigma$ bonds at the double bond):

$$
K_{\mathrm{AB}}^{\mathrm{PS} 2}(k, l, \ldots)=K_{\mathrm{AB}}-K_{\mathrm{AB}}^{\mathrm{froz}}[k, l, \ldots]
$$

and terms describing the interaction between two or more groups of orbitals as, e.g.,

$$
\begin{aligned}
K_{\mathrm{AB}}^{\mathrm{PS} 2}\left(k, k^{\prime} \leftrightarrow l\right)= & K_{\mathrm{AB}}-K_{\mathrm{AB}}^{\mathrm{froz}}\left[k, k^{\prime}\right]-K_{\mathrm{AB}}^{\mathrm{froz}}[l] \\
& +K_{\mathrm{AB}}^{\mathrm{froz}}\left[k, k^{\prime}, l\right] .
\end{aligned}
$$

It should be noted that the J-OC-PSP1 analysis can be analogously performed in terms of orbital groups (e.g., core, $\sigma$ bonds, $\pi$ bonds, lone pairs,...) rather than individual orbitals. 
The J-OC-PSP2 terms can be decomposed into an active and a passive contribution. For instance,

$$
\begin{aligned}
& K_{\mathrm{AB}}^{\mathrm{PSP} 2}(k)=K_{\mathrm{AB}}^{\mathrm{PSP} 2 \text { act }}(k)+K_{\mathrm{AB}}^{\mathrm{PSP} 2 \text { pass }}(k), \\
& K_{\mathrm{AB}}^{\mathrm{PSP} 2 \text { act }}(k)=K_{\mathrm{AB}}-K_{\mathrm{AB}}^{\mathrm{pass}}[k], \\
& K_{\mathrm{AB}}^{\mathrm{PSP} 2 \text { pass }}(k)=K_{\mathrm{AB}}^{\mathrm{pass}}[k]-K_{\mathrm{AB}}^{\text {froz }}[k] .
\end{aligned}
$$

$K_{\mathrm{AB}}^{\mathrm{PSP} 2 \text {,act }}(k \leftrightarrow l \leftrightarrow \cdots)$ contains all orbital paths where $k, l, \ldots$, all make an active contribution. Clearly, this term vanishes if any of the involved orbitals or orbital groups is passive or the J-OC-PSP2 term contains three or more orbitals.

The active $\mathbf{J}-\mathbf{O C}-\mathrm{PSP} 2$ terms are related to the $\mathrm{J}-\mathbf{O C}-$ PSP1 terms by Eqs. (30):

$$
\begin{aligned}
& K_{\mathrm{AB}}^{\mathrm{PS} 2, \mathrm{act}}(k)=K_{\mathrm{AB}}^{\mathrm{PSP} 1}(k)+\sum_{l, l \neq k} K_{\mathrm{AB}}^{\mathrm{PSP} 1}(k \leftrightarrow l), \\
& K_{\mathrm{AB}}^{\mathrm{PS} 2, \mathrm{act}}(k \leftrightarrow l)=K_{\mathrm{AB}}^{\mathrm{PS} 1}(k \leftrightarrow l) .
\end{aligned}
$$

Echo effects in spin-spin coupling: The two- and threeorbital J-OC-PSP2 terms can be linked to specific processes in the spin-spin coupling mechanism. An important kind of processes contained in the $K_{\mathrm{AB}}^{\mathrm{PSP} \text {,act }}(k \leftrightarrow l)$ term are orbital interactions that we call echoes: If orbital $k$ is perturbed by nucleus $\mathrm{B}$, the changes in orbital $k$ will result in changes in orbital $l$ as well. These changes in $l$ can in turn give rise to changes in $k$ in addition to those caused by nucleus B. Pictorially speaking, if all orbitals in the system are active or passive, orbital $k$ can be considered as supported by a system of springs symbolizing the interactions with the surrounding orbitals. If one or more of the surrounding orbitals are frozen, the springs are replaced by a rigid support, and $k$ will react differently to a external perturbation.

An example for an echo effect is the interaction of $\sigma$ and $\pi$ orbital in a multiple CC bond: If the $\sigma$ orbital is spin polarized by the perturbing nucleus or otherwise, it will induce an equally oriented spin polarization in the $\pi$ bond orbital. This spin polarization, in turn, will feed back into the $\sigma$ orbital and enhance its spin polarization. J-OC-PSP2 can detect this effect whereas J-OC-PSP1 cannot as the $\pi$ orbital is passive anyway. Another example for an echo effect is encountered by the $\mathrm{CH}$ bond orbitals around a perturbing $\mathrm{C}$ nucleus for the $\mathrm{CC}$ coupling in ethylene. The $\sigma(\mathrm{CC})$ orbital spin polarizes the $\sigma(\mathrm{CH})$ orbitals, which in turn modify the spin polarization of the $\sigma(\mathrm{CC})$ bond orbital.

The three-orbital terms $K_{\mathrm{AB}}^{\mathrm{PSP} 2}(k \leftrightarrow l \leftrightarrow m)$ correspond to a different coupling mechanism, viz. the spin transport by a passive orbital $l$ from an orbital $k$ via $l$ to a third orbital $m$. A typical example for a spin transport effect is given by the orbital path $1 s(\mathrm{C} 1) \rightarrow \pi(\mathrm{C} 1 \mathrm{C} 2) \rightarrow 1 s(\mathrm{C} 2)$ for the $\mathrm{C}-\mathrm{C}$ coupling constant in ethylene.

\section{B. Comparison of J-OC-PSP with other methods}

Perturbation-theoretical calculations with selected orbitals frozen have been used earlier to estimate the $\pi$ contribution to SSCCs. Contreras and co-workers ${ }^{33,34}$ performed calculations with frozen $\pi$ orbitals both for finite-perturbationtheory and self-consistent-perturbation theory at the intermediate neglect of differential overlap (INDO) level of theory. The basic idea of their approach is equivalent to ours. However, the methods presented by Contreras and co-workers $^{33,34}$ depend on the use of canonical MOs and allow only to freeze the $\pi$ system as a whole rather than individual $\pi$ orbitals or individual $\sigma$ orbitals in addition to the $\pi$ system. The same holds for the INDO- and sum-overstates (SOS)-2 calculations of $\pi$ contributions by Fukui and co-workers. ${ }^{35,36}$ Furthermore, the approach by Fukui and co-workers ${ }^{35,36}$ is based on a direct manipulation of the orbital Hessian, which requires that the Hessian is explicitly available. As was discussed in connection with Eqs. (5)(12), this is not the case for CP-DFT calculations. Hence, the algorithm presented here is more flexible and applicable to a wider range of computational levels than the methods discussed in Refs. 33-36.

\section{Implementation of the J-OC-PSP2 analysis: Summary of orbital terms}

From the theoretical derivation given in Sec. II A, a number of differently defined orbital contributions results, which have to be classified according to their importance and physical meaning. This is best done by orbital paths and the procedure outlined in the following. Actually, an orbital path and its contribution to the spin-spin coupling mechanism are of little relevance for the SSCC analysis (there exists an infinite number of orbital paths and it would be rather difficult to analyze the most important orbital paths individually), they help to understand the various orbital contributions, which comprise several (or an infinite number of) paths. (Note that an orbital path can contribute to more than one orbital contribution in J-OC-PSP2.) This can be clarified by considering the 12 orbital paths of Fig. 1. Paths 1, 2, and 3 are the first three members of an infinitely large set of paths defined by repeated involvement of orbital $k$. For example, path 2 describes how orbital $k$ is perturbed by the spin moment of nucleus B. This leads to a change in the exchange potential associated with $k$, which in turn changes $k$ again by self-exchange before orbital $k$ interacts with the spin moment of nucleus A. Hence, a path such as 1, 2, and 3 describe the self-consistent adjustment of orbital $k$ to the perturbation provided by nucleus B. Clearly, the self-consistent adjustment process is of little interest for the analysis and therefore it is reasonable to sum all the corresponding path contributions into a one-orbital term.

In Tables I, II, and III, we give an overview of the orbital contributions defined in this work. They are given for a nontrivial $\operatorname{SSCC}{ }^{1} \mathrm{~J}(\mathrm{~A}, \mathrm{~B})$ in a three-orbital system (orbitals $k, l$, $m$ ), for which 12 orbital paths (out of an infinite number) are shown in Fig. 1 to clarify the difference between the individual orbital contributions. Table I gives in its first two parts the few orbital contributions needed for the J-OC-PSP1 or J-OC-PSP2 analysis. Note that (a) the same orbital path can contribute to different orbital terms in J-OC-PSP2 and (b) the assignment of orbital paths to orbital contributions is handled essentially differently for J-OC-PSP1 (only active orbitals can carry an orbital contribution, and in the oneorbital contributions, they must be active at both A and B) and J-OC-PSP2 (any contribution, active or passive, of a 


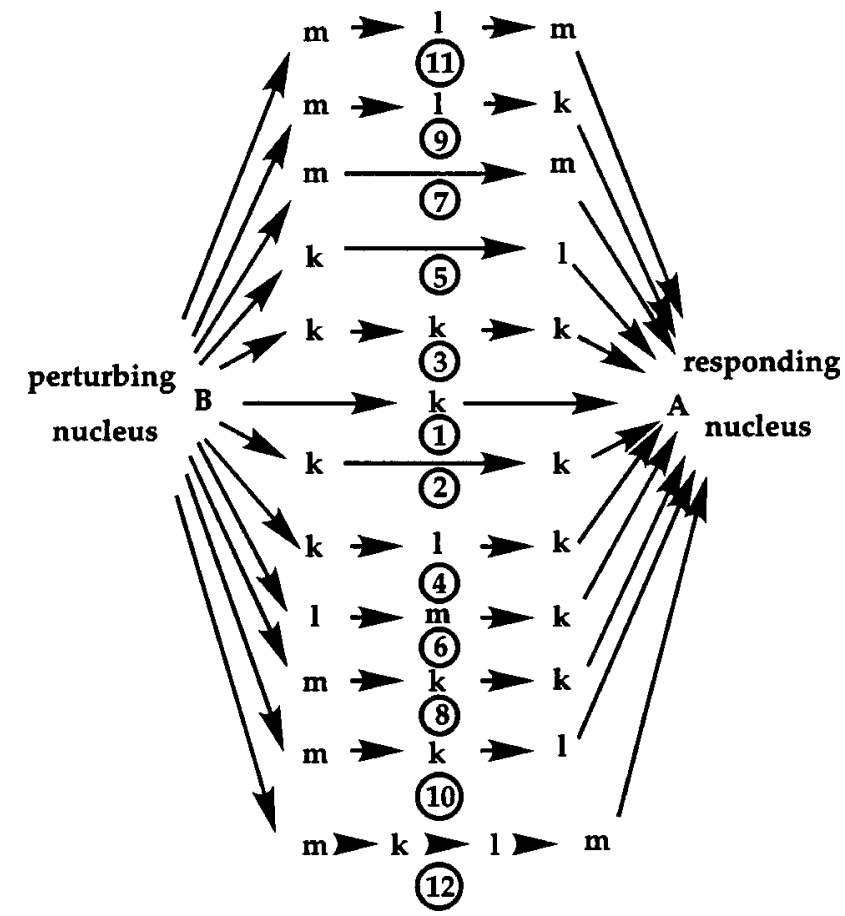

FIG. 1. Selected orbital paths for spin-spin coupling between perturbing nucleus $\mathrm{B}$ and responding nucleus $\mathrm{A}$ in a three-orbital system (orbitals $k, l$, $m$ ). The paths are numbered from 1 to 12 (encircled numbers).

given orbital, is summed into the one-orbital contribution of this orbital, see Table I). All the remaining orbital terms (third part of Table I) are auxiliary terms needed for the calculation rather than the analysis. The J-OC-PSP analyses can be drastically simplified if groups of orbitals rather than individual orbitals are considered. The corresponding orbital contributions are listed in Table II.

In Table III we have summarized the orbital contributions calculated by the NJC methods ${ }^{39,40}$ (classification according to the last orbital in the path; first part of Table I). Any analysis method that considers just the last orbital interacting with the responding nucleus ignores the major part of the information on the spin-spin coupling mechanism (both the active orbital at the perturbing nucleus and all intermediate steps) and is asymmetric with respect to the two coupling nuclei. In addition, any explanation must be done in terms of one-orbital contributions, which does not provide a basis to discuss the important steric exchange interactions. Therefore, we will not any longer consider terms of the type $K^{k}$ used in the NJC-methods. ${ }^{39,40}$ The same applies to an analysis based on Eq. (15) (second part of Table III) although such an analysis includes one-orbital terms $K^{(k)}$ and twoorbital terms $K^{(k \leftarrow l)}$ and therefore should be better suited for a discussion of the electronic effects influencing the spinspin coupling mechanism. However, any approach based on Eq. (15) suffers from the fact that this classification is based on the last two orbitals in the path. This is chemically misleading and breaks the symmetry between perturbing and responding nucleus unless just one- and two-bond SSCCs are considered. Hence, we will also refrain from calculating contributions $K^{(k)}$ and $K^{(k \leftarrow l)}$ (see Table III).

For a decomposition of the SSCC and its Ramsey terms into one- and two-orbital terms one must use J-OC-PSP1 and calculate $K^{\mathrm{PSP} 1}(k)$ and $K^{\mathrm{PSP} 1}(k \leftrightarrow l)$. This analysis can be improved by obtaining active and passive contributions

TABLE I. Distribution of different orbital paths among orbital contributions as described by J-OC-PSP1 and J-OC-PSP2 for a three-orbital problem. ${ }^{a}$

\begin{tabular}{|c|c|c|c|c|c|c|c|c|c|c|c|c|c|c|}
\hline \multirow[b]{2}{*}{ Type } & \multirow[b]{2}{*}{ Contribution } & \multirow[b]{2}{*}{ Eq. } & \multicolumn{12}{|c|}{ Orbital path included } \\
\hline & & & 1 & 2 & 3 & 4 & 5 & 6 & 7 & 8 & 9 & 10 & 11 & 12 \\
\hline \multicolumn{15}{|c|}{ J-OC-PSP1 contributions } \\
\hline \multicolumn{15}{|c|}{ Classification according to first and last orbital in path } \\
\hline One orbital & $K^{\mathrm{PSP} 1}(k)$ & $(21)$ & $\times$ & $\times$ & $\times$ & $\times$ & & & & & & & & \\
\hline Two orbital & $K^{\mathrm{PSP} 1}(k \leftrightarrow l)$ & $(22)$ & & & & & $\times$ & $\times$ & & & & & & \\
\hline \multirow{2}{*}{\multicolumn{15}{|c|}{$\begin{array}{l}\text { J-OC-PSP2 contributions } \\
\text { Classification according to all orbitals in path }\end{array}$}} \\
\hline & & & & & & & & & & & & & & \\
\hline One orbital & $K^{\mathrm{PSP} 2}(k)$ & $(25)$ & $\times$ & $x$ & $\times$ & $\times$ & $\times$ & $\times$ & & $\times$ & $\times$ & $\times$ & & $x$ \\
\hline active & $K^{\mathrm{PSP} 2, \text { act }}(k)$ & (29b) & $\times$ & $x$ & $\times$ & $\times$ & $\times$ & $\times$ & & $\times$ & $\times$ & & & \\
\hline passive & $K^{\mathrm{PSP} 2 \text {,pass }}(k)$ & $(29 c)$ & & & & & & & & & & $x$ & & $x$ \\
\hline Two orbital & $K^{\mathrm{PSP} 2}(k \leftrightarrow l)$ & (26a) & & & & $x$ & $x$ & $x$ & & & $x$ & $x$ & & $x$ \\
\hline Three orbital & $K^{\mathrm{PSP} 2}(k \leftrightarrow l \leftrightarrow m)$ & (26b) & & & & & & $\times$ & & & $x$ & $\times$ & & $\times$ \\
\hline \multicolumn{15}{|c|}{$\begin{array}{l}\text { Selected-orbital SSCC values required as auxiliary values } \\
\text { for the calculation of J-OC-PSP contributions }\end{array}$} \\
\hline All orbital & $K$ & (19) & $\times$ & $x$ & $\times$ & $\times$ & $\times$ & $\times$ & $x$ & $\times$ & $x$ & $x$ & $x$ & $x$ \\
\hline One orbital & $K^{\text {pass }}[\neg k]$ & (19) & $x$ & $x$ & $\times$ & $\times$ & & & & & & & & \\
\hline One orbital & $K^{\mathrm{pass}}[\neg(k, l)]$ & (19) & $\times$ & $x$ & $\times$ & $\times$ & $x$ & $\times$ & & & & & & \\
\hline$N-1$ orbital & $K^{\text {pass }}[k]$ & (19) & & & & & & & $x$ & & & $x$ & $x$ & $x$ \\
\hline One orbital & $K^{\text {froz }}[\neg k]$ & (23) & $\times$ & $x$ & $\times$ & & & & & & & & & \\
\hline$N-1$ orbital & $K^{\mathrm{froz}}[k]$ & $(23)$ & & & & & & & $x$ & & & & $x$ & \\
\hline$N-2$ orbital & $K^{\text {froz }}[k, l]$ & (23) & & & & & & & $x$ & & & & & \\
\hline$N-3$ orbital & $K^{\text {froz }}[k, l, m]$ & (23) & & & & & & & & & & & & \\
\hline
\end{tabular}

${ }^{a}$ The numbering of the orbital paths refers to Fig. 1. An $\times$ in a table entry indicates that the orbital contribution shown in the corresponding row includes the orbital path given in the corresponding column. 
TABLE II. Distribution of different orbital paths among orbital contributions as described by J-OC-PSP1 and J-OC-PSP2 for a three-orbital problem: Simplified version based on orbital groups. ${ }^{\text {a }}$

\begin{tabular}{|c|c|c|c|c|c|c|c|c|c|c|c|c|c|c|}
\hline \multirow[b]{2}{*}{ Type } & \multirow[b]{2}{*}{ Contribution } & \multirow[b]{2}{*}{ Eq. } & \multicolumn{12}{|c|}{ Orbital path included } \\
\hline & & & 1 & 2 & 3 & 4 & 5 & 6 & 7 & 8 & 9 & 10 & 11 & 12 \\
\hline \multicolumn{15}{|c|}{ J-OC-PSP1 contributions } \\
\hline One orbital & $K^{\mathrm{PSP} 1}(k, l)$ & & $\times$ & $\times$ & $x$ & $\times$ & $x$ & $x$ & & & & & & \\
\hline Two orbital & $K^{\mathrm{PSP} 1}(k \leftrightarrow l, m)$ & & & & & & $\times$ & $\times$ & & $\times$ & $\times$ & & & \\
\hline \multicolumn{15}{|c|}{ J-OC-PSP2 contributions } \\
\hline One orbital & $K^{\mathrm{PSP} 2}(k, l)$ & (27) & $\times$ & $\times$ & $\times$ & $x$ & $\times$ & $\times$ & & $\times$ & $x$ & $\times$ & $\times$ & $x$ \\
\hline Two orbital & $K^{\mathrm{PSP} 2}(k \leftrightarrow l, m)$ & (28) & & & & $x$ & $\times$ & $\times$ & & $\times$ & $\times$ & $\times$ & & $\times$ \\
\hline
\end{tabular}

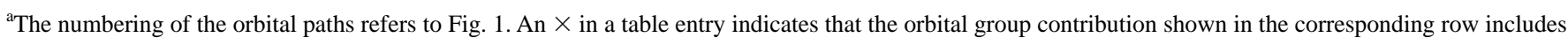
the orbital path given in the corresponding column.

and higher order orbital terms at the J-OC-PSP2 (see Table I; at the J-OC-PSP1 level only active orbital contributions are considered) and thus only active orbitals can have nonzero orbital contributions, whereas orbital contributions at the J-OC-PSP2 level contain all contributions, no matter whether active or passive, of this orbital to the spin-spin coupling. In the latter case a number of auxiliary orbital contributions obtained in selected orbital SSCC calculations is needed to derive the actual orbital contributions $K^{\mathrm{PSP} 2}(k)$ and $K^{\mathrm{PSP} 2}(k \leftrightarrow l)$. Hence, for the analysis only two different types of orbital contributions (four if active and passive contributions must be considered) are required whereas the calculations must also consider terms of the type $K[k], K[k, l]$, etc. (see Table I). This comparison shows that although more than 20 different orbital contributions have been defined in this work, only a few are of importance for the actual J-OC-PSP analysis.

When applying the J-OC-PSP methods to, e.g., to the SSCC ${ }^{1} \mathrm{~J}(\mathrm{CC})$ of ethylene, the relevant orbital contributions are $K^{\mathrm{PSP} 1}(\sigma(\mathrm{CC}))$ and $K^{\mathrm{PSP} 1}(\sigma(\mathrm{CH}))$, where at the $\mathrm{J}-\mathrm{OC}-$ PSP2 level also the corresponding passive contribution of the $\pi$ orbital is obtained. For the simple analysis it is sufficient to handle all $\sigma(\mathrm{CH})$ orbitals in a single group thus reducing the number of calculations to just three. In this work, however, we will further detail into individual $\sigma(\mathrm{CH})$ contributions to clarify, which terms are important and which are not.
For the calculation of the auxiliary terms $K[k], K[k, l]$, etc., given in Table I, the existing CP-DFT program ${ }^{16}$ was extended. By performing the selective orbital calculations in an order given by the equations in Sec. II A, the active and passive contributions of Table I are obtained.

\section{Computational details}

All SSCC calculations were carried out with the CPDFT method described previously by us. ${ }^{16}$ For this purpose, the hybrid functional B3LYP ${ }^{41-43}$ and the basis set $(11 s, 7 p, 2 s / 6 s, 2 p) /[7 s, 6 p, 2 d / 4 s, 2 p]$ (Refs. 44 and 45$)$ designed for the calculation of magnetic properties was used. The SSCCs of ethylene were calculated at the experimental geometry of the molecule from Ref. 46 (bond lengths and bond angles are given in scheme 1).

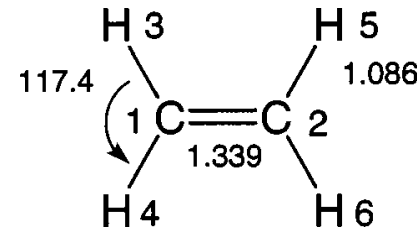

The J-OC-PSP analysis and the orbital-selected SSCC calculations were carried out for LMOs obtained with a Boys localization ${ }^{47}$ where however core, $\sigma$, and $\pi$ orbitals are

TABLE III. Comparison with other analysis methods. ${ }^{a}$

\begin{tabular}{|c|c|c|c|c|c|c|c|c|c|c|c|c|c|c|}
\hline \multirow[b]{2}{*}{ Type } & \multirow[b]{2}{*}{ Contribution } & \multirow[b]{2}{*}{ Eq. } & \multicolumn{12}{|c|}{ Orbital path included } \\
\hline & & & 1 & 2 & 3 & 4 & 5 & 6 & 7 & 8 & 9 & 10 & 11 & 12 \\
\hline \multicolumn{15}{|c|}{ Simple post-processing decomposition } \\
\hline \multicolumn{15}{|c|}{ Classification according to last orbital in path } \\
\hline One orbital & $K^{k}$ & $(13)$ & $\times$ & $x$ & $\times$ & $x$ & & $x$ & & $x$ & $\times$ & & & \\
\hline \multicolumn{15}{|c|}{ Decomposition based on $\widetilde{F}_{l}^{(B), X}$} \\
\hline \multicolumn{15}{|c|}{ Classification according to last two orbitals in path } \\
\hline One orbital & $K^{(k)}$ & $(15 c)$ & $x$ & $x$ & $x$ & & & & & $x$ & & & & \\
\hline Two orbital & $K^{(k \leftarrow l)}$ & $(15 \mathrm{~d})$ & & & & $\times$ & & & & & $\times$ & & & \\
\hline Two orbital & $K^{(l \leftarrow k)}$ & $(15 d)$ & & & & & $x$ & & & & & $\times$ & & \\
\hline Two orbital & $K^{(l \mapsto k)}$ & $(15 \mathrm{e})$ & & & & $x$ & $\times$ & & & & $x$ & $\times$ & & \\
\hline
\end{tabular}

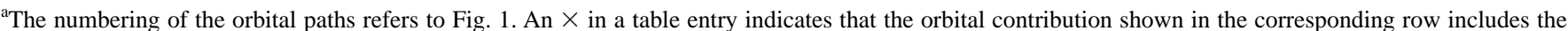
orbital path given in the corresponding column. 
TABLE IV. Ramsey terms, their total $\pi$ total, and their active $\pi$ contributions for all SSCCs of ethylene. ${ }^{\text {a }}$

\begin{tabular}{|c|c|c|c|c|c|c|c|c|c|c|c|c|c|c|c|c|c|}
\hline \multirow{3}{*}{$\begin{array}{l}\text { Coupling } \\
\text { nuclei }\end{array}$} & \multicolumn{4}{|c|}{$\mathrm{FC}$} & \multicolumn{4}{|c|}{ PSO } & \multicolumn{3}{|c|}{ DSO } & \multicolumn{4}{|c|}{ SD } & \multicolumn{2}{|c|}{ Total } \\
\hline & \multirow[b]{2}{*}{ Total } & \multicolumn{2}{|c|}{ Total $\pi$} & \multirow[b]{2}{*}{ Act $\pi$} & \multirow[b]{2}{*}{ Total } & \multicolumn{2}{|c|}{ Total $\pi$} & \multirow[b]{2}{*}{ Act $\pi$} & \multirow[b]{2}{*}{ Total } & \multirow[b]{2}{*}{ Total $\pi$} & \multirow[b]{2}{*}{ Act $\pi$} & \multirow[b]{2}{*}{ Total } & \multicolumn{2}{|c|}{ Total $\pi$} & \multirow[b]{2}{*}{ Act $\pi$} & \multirow[b]{2}{*}{ CP-DFT } & \multirow[b]{2}{*}{ Expt. } \\
\hline & & $\mathrm{Hz}$ & SI & & & $\mathrm{Hz}$ & SI & & & & & & $\mathrm{Hz}$ & SI & & & \\
\hline $\mathrm{C} 1-\mathrm{C} 2$ & 79.1 & 4.5 & 5.9 & 0.0 & -10.1 & -2.2 & -2.9 & -2.2 & 0.1 & $0.0_{4}$ & $0.0_{4}$ & 3.7 & 4.6 & 2.8 & 4.6 & 72.8 & 67.6 \\
\hline $\mathrm{H} 3-\mathrm{C} 1$ & 153.4 & 2.9 & 1.0 & 0.0 & 0.5 & 0.8 & 0.3 & 0.8 & 0.4 & 0.3 & 0.3 & 0.1 & 0.4 & 0.1 & 0.4 & 154.4 & 156.4 \\
\hline $\mathrm{H} 3-\mathrm{C} 2$ & 0.6 & -2.4 & -0.8 & 0.0 & -1.1 & -0.3 & -0.1 & -0.3 & -0.7 & $-0.0_{3}$ & $-0.0_{3}$ & 0.0 & 0.0 & 0.0 & 0.0 & -1.2 & -2.4 \\
\hline $\mathrm{H} 3-\mathrm{H} 4$ & 1.9 & -1.5 & -0.2 & 0.0 & 4.1 & 0.6 & 0.0 & 0.6 & -3.8 & -0.1 & -0.1 & 0.3 & 0.1 & 0.0 & 0.1 & 2.5 & 2.5 \\
\hline $\mathrm{H} 3-\mathrm{H} 5$ & 11.9 & 1.5 & 0.2 & 0.0 & 0.7 & 0.1 & 0.0 & 0.1 & -1.1 & $-0.0_{4}$ & $-0.0_{4}$ & -0.1 & -0.1 & 0.0 & -0.1 & 11.4 & 11.7 \\
\hline $\mathrm{H} 3-\mathrm{H} 6$ & 18.5 & 1.5 & 0.2 & 0.0 & 3.2 & 0.4 & 0.0 & 0.4 & -3.6 & -0.3 & -0.3 & 0.3 & 0.4 & 0.0 & 0.4 & 18.4 & 19.1 \\
\hline
\end{tabular}

${ }^{a}$ The total $\pi$ contribution is given as the sum of active (act) and passive $\pi$ contribution. Experimental values from Ref. 35. For nuclei numbering, see scheme 1. All SSCCs are expressed in Hz, the total $\pi$ contribution is also given as reduced SSCC in SI units of $10^{19} \mathrm{~J} \mathrm{~T}^{-2}$ to facilitate comparison between different types of SSCCs. CP-DFT/B3LYP/ $(11 s, 7 p, 2 d / 5 s, 1 p)[7 s, 6 p, 2 d / 4 s, 2 p]$ calculations. The first nucleus of each pair is the perturbed nucleus.

separately localized for reasons described elsewhere. ${ }^{20}$ All discussions are based on LMOs.

For the visualizing of the FC coupling mechanism, we will show diagrams of the FC spin density distribution $\rho^{(B), \mathrm{FC}}(\mathbf{r})$ derived recently, ${ }^{20}$

$$
\rho^{(B), \mathrm{FC}}(\mathbf{r})=2 \sum_{k}^{\mathrm{occ}} \psi_{k}^{(0)}(\mathbf{r}) \psi_{k}^{(B), \mathrm{FC}}(\mathbf{r}) .
$$

The distribution $\rho^{(B), \mathrm{FC}}(\mathbf{r})$ can be split up into orbital contributions. The $\rho^{(B), \mathrm{FC}}(\mathbf{r})$ and selected orbital contributions will be represented in form of contour line plots, where the contour levels are given by a geometric progression with the ratio of $100^{1 / 5}$ between two subsequent contours. All SSCC calculations are performed with the $a b$ initio program package COLOGNE $2003 .{ }^{48}$

\section{INVESTIGATION OF THE $\pi$ MECHANISM IN ETHYLENE-THE ROLE OF PASSIVE ORBITALS}

In the following, we will discuss the results obtained for ethylene and unravel the role of the passive orbital contributions step by step. Our focus will be predominantly on the $\pi$ orbital and the spin information mediated by it. We will calculate the $\pi$ orbital contribution to the FC term and total SSCC.

\section{A. The $\pi$ spin-spin coupling mechanism in ethylene}

In Table IV, the calculated and measured SSCCs of ethylene are compared. In addition, the four Ramsey terms of the SSCCs are partitioned into active and passive $\pi$ contributions. The total $\pi$ contribution to each Ramsey term is given as well as the active part of this contribution. The passive $\pi$ contribution is obtained by deducting the active part from the total $\pi$ term. Similarly, one obtains the total $\sigma$ contributions by subtracting $\pi$ and core parts from the actual value of the Ramsey term. In Table V, the orbital contributions to $\sigma$ and $\pi$ part, calculated according to Eq. (13), are listed.

The calculated SSCCs of ethylene are in reasonable agreement with the measured ones ${ }^{4,5}$ (see also Refs. 33-36) where one has to consider that the latter must be adjusted for vibrational corrections to become directly comparable with the CP-DFT values. In this work, we are interested more in the observed trends of the SSCCs of a $\pi$ system rather than their absolute values and in this respect the obtained agreement between theory and experiment is satisfactory.

Although the FC term dominates all SSCCs, the other Ramsey terms are non-negligible (Table IV). It is however a fact that the three noncontact terms largely cancel each other out. Therefore, we focus first on the FC coupling mechanism and the involvement of the $\pi$ electrons in this mechanism. Clearly there cannot be an active contribution to the FC mechanism by the $\pi$ electrons (Table IV) because they possess zero density at the nuclei, which excludes any spin polarization of the $\pi$ density by the internal magnetic field of the nucleus. However, there is a passive contribution of the $\pi$ electrons to the $\mathrm{FC}$ term, which, despite the strong variation of the FC term between 0.6 and $153.4 \mathrm{~Hz}$, is rather constant (1.5 to $4.5 \mathrm{~Hz}$; Table IV). As a result of this, the importance of the $\pi$ part of the FC coupling mechanism increases with the length of the coupling path: For one-bond $\mathrm{FC}(\mathrm{CH})$ coupling the $\pi$ contribution is just $2 \%$ of the total FC term whereas for the three-bond FC terms it is more than $10 \%$. The geminal FC terms represent an exception in so far as the magnitude of the $\pi$ part is comparable or even larger than that of the actual FC term and their signs differ.

In Fig. 2, the total FC spin polarization distribution [Fig. 2(a)], the $\pi$ orbital contribution to the FC spin density [Fig. 2(b)] and the $\pi$-induced part of the FC spin density in $\sigma(\mathrm{C} 1 \mathrm{C} 2)$ shown for ethylene in the $y z$ plane, i.e., the plane cutting through the $\pi$ orbital of the molecule. The perturbing nucleus is $\mathrm{C} 1$ and since an $\alpha$-spin moment is assumed for the perturbing nucleus, Fermi coupling should imply a dominance of $\beta$ electron spin in the vicinity of $\mathrm{C} 1$. This is actually the case, but only in a small core region next to $\mathrm{C} 1$ (about $0.2 \AA$ around C1) not visible in Fig. 2(a). This core region is surrounded by a spherical region with a dominance of $\alpha$ spin [concentric solid circles in Fig. 2(a)] which in turn is surrounded by a sphere of $\beta$ spin. The region around $\mathrm{C} 1$ is separated from a large region of $\alpha$-spin dominance surrounding $\mathrm{C} 2$ by a nodal surface [Fig. 2(a)].

The portion of the FC spin polarization that belongs to the $\pi(\mathrm{CC})$ orbital is displayed in Fig. 2(b). This spin density is nearly identical with the spin density belonging to the $\mathrm{J}-\mathrm{OC}-\mathrm{PSP} 2$ contribution $\mathrm{FC}^{\mathrm{PSP} 2}[\pi(\mathrm{CC})]$ (not shown), i.e., the impact a freezing of the $\pi$ orbital has on the total spin density. In the following, we will use the notation $\operatorname{FC}(\pi)$ for 
TABLE V. Decomposition of the passive $\pi$ contribution to the FC term of the SSCCs of ethylene into orbitals transmitting the $\pi$ spin-spin coupling mechanism to the responding nucleus. ${ }^{\mathrm{a}}$

\begin{tabular}{|c|c|c|c|c|c|c|c|}
\hline & $1 s(\mathrm{C} 2)$ & $1 s(\mathrm{C} 1)$ & $\sigma(\mathrm{C} 2 \mathrm{H} 6)$ & $\sigma(\mathrm{C} 2 \mathrm{H} 5)$ & $\sigma(\mathrm{C} 1 \mathrm{C} 2)$ & $\sigma(\mathrm{C} 1 \mathrm{H} 4)$ & $\sigma(\mathrm{C} 1 \mathrm{H} 3)$ \\
\hline \multicolumn{8}{|l|}{$\mathrm{C} 1-\mathrm{C} 2$} \\
\hline FC(Tot) & -7.69 & -0.10 & -19.51 & -19.51 & 146.36 & -10.24 & -10.24 \\
\hline \multirow{2}{*}{$\mathrm{FC}(\pi)$} & -3.88 & 0 & 2.02 & 2.02 & 4.39 & -0.03 & -0.03 \\
\hline & -5.11 & 0 & 2.66 & 2.66 & 5.78 & -0.04 & -0.04 \\
\hline \multicolumn{8}{|l|}{$\mathrm{H} 3-\mathrm{C} 1$} \\
\hline $\mathrm{FC}(\mathrm{Tot})$ & 0 & -8.89 & -2.05 & 1.55 & -24.02 & -26.08 & 212.91 \\
\hline \multirow[t]{2}{*}{$\operatorname{FC}(\pi)$} & 0 & -2.56 & -0.01 & -0.01 & 2.42 & 1.35 & 1.75 \\
\hline & 0 & -0.85 & $-0.00_{4}$ & $-0.00_{3}$ & 0.80 & 0.45 & 0.58 \\
\hline \multicolumn{8}{|l|}{$\mathrm{H} 3-\mathrm{C} 2$} \\
\hline $\mathrm{FC}(\mathrm{Tot})$ & 2.80 & 0 & -7.61 & 2.28 & -0.23 & -0.84 & 4.17 \\
\hline \multirow[t]{2}{*}{$\operatorname{FC}(\pi)$} & 2.25 & 0 & -1.21 & -1.23 & -2.28 & $0.00_{1}$ & 0.03 \\
\hline & 0.74 & 0 & -0.40 & -0.41 & -0.75 & 0.00 & 0.01 \\
\hline \multicolumn{8}{|l|}{$\mathrm{H} 3-\mathrm{H} 4$} \\
\hline $\mathrm{FC}(\mathrm{Tot})$ & 0 & 0 & 0.21 & 0.16 & -0.44 & -0.99 & 2.95 \\
\hline \multirow[t]{2}{*}{$\mathrm{FC}(\pi)$} & 0 & 0 & $-0.00_{4}$ & -0.01 & 0.03 & -1.51 & 0.05 \\
\hline & 0 & 0 & 0.00 & 0.00 & $0.00_{2}$ & -0.13 & $0.00_{3}$ \\
\hline \multicolumn{8}{|l|}{ H3-H5 } \\
\hline $\mathrm{FC}(\mathrm{Tot})$ & 0 & 0 & 0.50 & 9.15 & -0.66 & 0.63 & 2.27 \\
\hline \multirow[t]{2}{*}{$\mathrm{FC}(\pi)$} & 0 & 0 & -0.03 & 1.54 & -0.03 & $0.00_{4}$ & $0.00_{3}$ \\
\hline & 0 & 0 & $-0.00_{2}$ & 0.13 & $-0.00_{2}$ & 0.00 & 0.00 \\
\hline \multicolumn{8}{|l|}{ H3-H6 } \\
\hline FC(Tot) & 0 & 0 & 13.62 & 0.39 & 0.64 & 0.61 & 3.24 \\
\hline \multirow[t]{2}{*}{$\mathrm{FC}(\pi)$} & 0 & 0 & 1.53 & -0.03 & -0.04 & $0.00_{4}$ & 0.01 \\
\hline & 0 & 0 & 0.13 & $-0.00_{2}$ & $-0.00_{3}$ & $0.00^{4}$ & 0.00 \\
\hline
\end{tabular}

${ }^{a}$ For nuclei numbering see Scheme 1. The nucleus given first is the perturbing nucleus. Italicized numbers are reduced SSCCs in SI units of $10^{19} \mathrm{~J} \mathrm{~T}^{-2}$, all others are in $\mathrm{Hz}$. CP-DFT/B3LYP/ $(11 s, 7 p, 2 d / 5 s, 1 p)$ $\times[7 s, 6 p, 2 d / 4 s, 2 p]$ calculations.

the $\mathrm{J}-\mathrm{OC}-\mathrm{PSP} 2$ contribution of the total $\pi$ system, i.e., we will write $\mathrm{FC}$ instead of $\mathrm{J}^{\mathrm{FC}}$ and omit the superscript PSP2, as all orbital analyses in the present work are done at the J-OC-PSP2 level.

As the $\pi(\mathrm{CC})$ orbital of ethylene has nodes at all atoms, this spin polarization is of a passive character being induced by the orbitals $1 s, \sigma(\mathrm{CC})$, and $\sigma(\mathrm{CH})$; similarly its impact on the responding nucleus needs to be mediated by other elec-
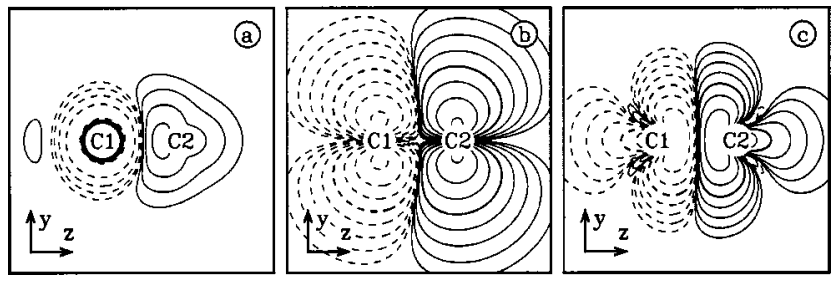

FIG. 2. The FC spin polarization for ethylene. The contour line diagrams are given for a plane through the $\mathrm{C} 1 \mathrm{C} 2$ bond perpendicular to the plane of the molecule. The drawing plane is considered as $y z$ plane, with the $z$ axis along the bond $\mathrm{C} 1 \mathrm{C} 2$. Contour levels were chosen in geometric progression with a ratio of $100^{1 / 5}$ between neighboring levels. For the purpose of facilitating a comparison of levels, the contours for 0.1 and 10 are shown in boldface. Solid (dashed) lines represent positive (negative) values of the FC spin density distribution, the dotted line is the zero contour. Calculations were done at the B3LYP/ $[7 s, 6 p, 2 d / 4 s, 2 p]$ level of theory. The perturbing nucleus is $\mathrm{C} 1$. (a) The total FC spin density distribution. (b) The contribution of the $\pi(\mathrm{C} 1 \mathrm{C} 2)$ orbital to the total FC spin density. Scaling factor for the contour levels is 100 . (c) The contribution of the $\sigma(\mathrm{C} 1 \mathrm{C} 2)$ orbital to the $\mathrm{FC}(\pi)$ spin density (scaled by 1000). trons. At $\mathrm{C} 1$, there is a dominance of $\beta$ spin and at $\mathrm{C} 2$ of $\alpha$ spin. This is also the case when considering just that part of the passive $\pi$ contribution to the FC spin polarization transported by the $\sigma(\mathrm{CC})$ orbital to the responding nucleus. This part is obtained as a FC spin density difference for the $\sigma(\mathrm{CC})$ orbital with the $\pi(\mathrm{CC})$ orbital kept once unfrozen (positive sign) and once frozen (negative sign). The spin polarization of the $\pi(\mathrm{CC})$ orbital [Fig. 2(b)] as well as its echo on the $\sigma(\mathrm{CC})$ orbital [Fig. 2(c)], is nearly antisymmetric. Without understanding the FC spin-spin coupling mechanism, it is no longer possible to tell whether $\mathrm{C} 1$ or $\mathrm{C} 2$ is the perturbing nucleus as the spin information is transferred to the $\pi$ orbital via other orbitals rather than from the nuclear magnetic moment.

Figures 2(b) and 2(c) suggest that the $\pi$ mechanism in ethylene should follow a simple pattern: From the perturbing nucleus, the spin information is coupled into the $\pi$ orbital via some $\sigma$ orbital or chain of $\sigma$ orbitals. The only possible reaction of the $\pi$ orbital is a polarization along the CC bond axis, and that results in a surplus of $\alpha$ density in the $\pi$ area around one of the $\mathrm{C}$ atoms and a corresponding $\beta$ surplus density at the other $\mathrm{C}$ atom in the bond. Hence, the values of the FC $(\pi)$ terms should be less dependent on details of the bonding situation than the value for the total FC term.

Figure 3 schematically shows the inclusion of the $\pi$ orbitals into the FC coupling mechanism in the case of ${ }^{1} J(\mathrm{CC})$. The density of the $1 s(\mathrm{C} 1), \sigma(\mathrm{C} 1 \mathrm{C} 2), \sigma(\mathrm{C} 1 \mathrm{H} 3)$, and 

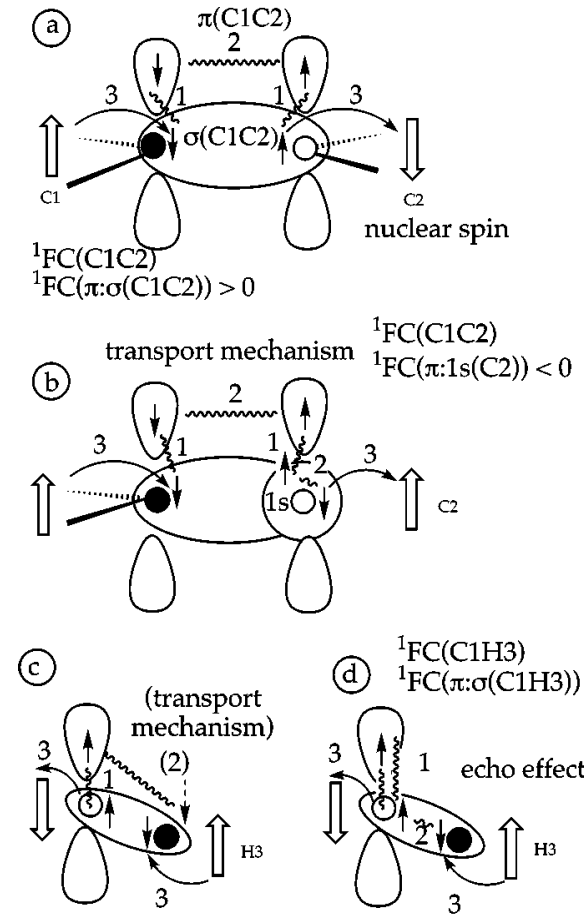

(d) ${ }^{1} \mathrm{FC}(\pi: \sigma(\mathrm{C} 1 \mathrm{H} 3))>0$

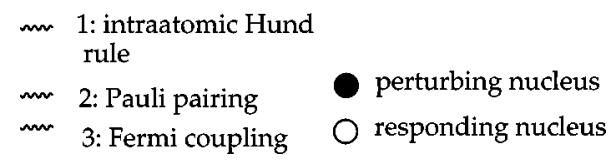

FIG. 3. FC coupling mechanism in ethylene involving the passive $\pi$ orbital. (a) The $\sigma(\mathrm{C} 1 \mathrm{C} 2)$ contribution to $\mathrm{FC}(\pi)$ of the ${ }^{1} \mathrm{FC}(\mathrm{C} 1 \mathrm{C} 2)$ coupling. For the perturbing nucleus $\mathrm{C} 1$ an $\alpha$ spin moment is assumed (large open arrow). The small arrows indicate the $\alpha$ (up) and $\beta$ (down) spin. The curved arrows denote Fermi coupling (3). The exchange interactions leading to spin polarization (wiggle lines) between the electrons are schematically given for intra-atomic exchange optimization [intra-atomic Hund rule (1)] and Pauli pairing (2). (b) The $1 s(\mathrm{C} 2)$ contribution to $\mathrm{FC}(\pi)$ of the ${ }^{1} \mathrm{FC}(\mathrm{C} 1 \mathrm{C} 2)$ coupling. (c) The $\sigma(\mathrm{C} 1 \mathrm{CH} 3)$ contribution to $\mathrm{FC}(\pi)$ of the ${ }^{1} \mathrm{FC}(\mathrm{C} 1 \mathrm{H} 3)$ coupling. Transport mechanism caused by the anisotropy of the $\mathrm{CH}$ bond density. A dashed arrow indicates partial electron spin. (d) The $\sigma(\mathrm{C} 1 \mathrm{CH} 3)$ contribution to $\mathrm{FC}(\pi)$ of the ${ }^{1} \mathrm{FC}(\mathrm{C} 1 \mathrm{H} 3)$ coupling. Echo effect caused by the passive $\pi$ orbital. For (c) and (d) H3 is the perturbing nucleus.

$\sigma(\mathrm{C} 1 \mathrm{H} 4)$ orbital is spin polarized by the perturbing nucleus $\mathrm{C} 1$ by Fermi coupling [Fig. 3(a), mechanism 3] in the way that the spin density close to $\mathrm{C} 1$ is dominated by $\beta$ spin. The spin-polarized $\sigma$ density causes also a dominance of $\beta$ spin in the $\pi$ orbital at $\mathrm{C} 1$ according to the intra-atomic Hund rule (mechanism 1). Pauli coupling (mechanism 2) of the electrons of the $\pi$ bond leads to a dominance of $\alpha$ spin at nucleus $\mathrm{C} 2$, which of course does not affect the spin of nucleus $\mathrm{C} 2$, however the $\mathrm{C} 2$ surrounding $\sigma$ density is affected in the way that this also is preferentially of $\alpha$ spin (mechanism 1). The $\sigma$-spin density leads to a $\beta$-spin moment of nucleus $\mathrm{C} 2$ (Fermi coupling, 3) and a positive $\pi$ contribution of $4.4 \mathrm{~Hz}$, i.e., by this amount the FC coupling mechanism transmitted through the $\sigma(\mathrm{CC})$-spin density $(142 \mathrm{~Hz}$, Table $\mathrm{V})$ is increased.

The external $\mathrm{CH}$ bonds all lead to a negative $\sigma$ contribution to ${ }^{1} \mathrm{FC}(\mathrm{CC})(-21.5$ and $-10.2 \mathrm{~Hz}$, Table $\mathrm{V})$, which is typical of one-bond coupling constants and has been discussed in Ref. 20 (the one-bond path is extended to a twobond coupling path thus implying a change in sign). At the site of the responding nucleus $\mathrm{C} 2$, there are passive $\pi$ contributions of $2.0 \mathrm{~Hz}$ (Table V) caused by a transmission of the $\pi$-spin polarization to the $\sigma(\mathrm{C} 2 \mathrm{H} 5)$ - and $\sigma(\mathrm{C} 2 \mathrm{H} 6)$-spin density. Since the mechanism via the $\pi$-orbital functions in the same way as described in Fig. 3(a), the contribution is positive, although somewhat smaller than for $\sigma(\mathrm{C} 1 \mathrm{C} 2)$ considering the fact that the $\mathrm{CH}$ LMOs have smaller amplitudes at the responding nucleus.

A relatively strong passive $\pi$ contribution of $-3.9 \mathrm{~Hz}$ is obtained via the $1 s$ core orbital at $\mathrm{C} 2$. This change in sign and the FC spin density pattern at $\mathrm{C} 1$ are related. The $1 \mathrm{~s}$ orbital is fully localized in the core region of $\mathrm{C} 2$. If the electron in the outer sphere of the $1 s(\mathrm{C} 2)$ orbital adopts preferentially $\alpha$ spin because of the spin-polarization mechanism via the $\pi$ orbital [Fig. 3(b)], the $\beta 1 s$ electron must contract toward the $\mathrm{C} 2$ nucleus and generate a surplus of $\beta$ spin there [Fig. 3(b)], as $\alpha$ and $\beta$ electron always respond in an opposite way to the FC perturbation. Fermi coupling leads to an $\alpha$ nuclear spin and accordingly to a negative $1 s(\mathrm{C} 2)$ contribution of the passive $\pi$ part.

The electron pair in the $1 s(\mathrm{C} 1)$ orbital is spin polarized in the opposite way ( $\beta$ spin close to the nucleus; $\alpha$ spin in the outer sphere of the $1 s$ orbital) as can be seen in Fig. 3(b). This spin distribution is superimposed by that of the $s p^{2}$ hybrid orbital forming the CC $\sigma$ bond, which is completely negative ( $\beta$ dominated) in the region of $\mathrm{C} 1$ but positive $(\alpha$ dominated) in the region of $\mathrm{C} 2$ again due to the opposite response of $\alpha$ and $\beta$ electron, which is enhanced by exchange effects and left-right correlation. Figure 2(a) shows that in the (outer) core region of $\mathrm{C} 1$ the spin polarization of $1 s$ (dominance of $\alpha$ ), in the valence region the spin polarization of the $s p^{2}$ hybrid orbital dominates (dominance of $\beta$ ).

The passive $\pi$ contributions to the FC term of ${ }^{1} \mathrm{~J}(\mathrm{C} 1 \mathrm{H} 3)$ can be explained in the same way if one considers that the $\sigma$-bond density $\mathrm{C} 1 \mathrm{H} 3$ is anisotropic (because of the $\pi$ density at $\mathrm{C} 1$ ) rather than rotationally symmetric. In the quantum chemical calculation, this can only be correctly described by the inclusion of $p$-type polarization functions at the $\mathrm{H}$ atom. Hence, there is a $\pi$-type contribution to reflect the anisotropy of the $\mathrm{C} 1 \mathrm{H} 3$ bond density. In Figs. 3(c) and 3(d), this is schematically indicated where Fig. 3(c) indicates the $\pi$-type mechanism similar to the case of ${ }^{1} \mathrm{~J}(\mathrm{CC})$ [Fig. 3(a)] and Fig. 3(d) describes an echo effect: spin polarization is transmitted via the $\sigma(\mathrm{C} 1 \mathrm{H} 3)$-orbital to nucleus $\mathrm{C} 1(\mathrm{H} 3$ is the perturbing nucleus). There it causes the spin polarization of the $\pi$ density at $\mathrm{C} 1$, which can in turn increase the spin polarization of any s-type density close to the nucleus $\mathrm{C} 1$.

Clearly, the $\pi$ effects for the ${ }^{1} \mathrm{FC}(\mathrm{CH})$ coupling mechanism are smaller than those of the ${ }^{1} \mathrm{FC}(\mathrm{CC})$ one as there are no real $p$ orbitals at the $\mathrm{H}$ atom. However, both the type and the relative ratio of positive and negative $\pi$ contributions are the same so that the calculated value of $2.9 \mathrm{~Hz}$ reflects again the partial cancellation of positive valence $(2.4,1.7,1.4 \mathrm{~Hz}$, Table V) and the negative core contribution $(-2.8 \mathrm{~Hz})$.

All passive $\pi$-contributions to the orbital terms listed in Table V can be explained in a similar way. Only those orbitals with a sizable amplitude at the responding nucleus lead to a significant contribution. For example, for the $\mathrm{C} 1 \mathrm{C} 2$ coupling, these are the $\sigma(\mathrm{C} 1 \mathrm{C} 2), \sigma(\mathrm{C} 2 \mathrm{H} 5), \sigma(\mathrm{C} 2 \mathrm{H} 6)$, and the 
TABLE VI. Comparison of the passive $\pi$ contributions to the FC term for the SSCCs of ethylene as calculated with different methods. ${ }^{\mathrm{a}}$

\begin{tabular}{lcrrrrrrr}
\hline \hline & & \multicolumn{2}{c}{ INDO/SOS2 } & & \multicolumn{3}{c}{ CP-DFT/B3LYP } & Expt. \\
\cline { 3 - 4 } \cline { 6 - 8 } Nuclei & \# Bonds & \multicolumn{1}{c}{ FC } & FC $(\pi)$ & & FC & FC $(\pi)$ & J & J \\
\hline $1-2$ & 1 & 82.1 & 15.0 & & 79.1 & 4.5 & 72.8 & 67.6 \\
$3-1$ & 1 & 156.7 & 4.4 & & 153.5 & 2.9 & 154.4 & 156.4 \\
$3-2$ & 2 & -11.6 & -4.4 & & 0.6 & -2.4 & -1.2 & -2.4 \\
$3-4$ & 2 & 3.2 & -1.3 & & 1.9 & -1.5 & 2.5 & 2.5 \\
$3-5$ & 3 & 9.3 & 1.3 & & 11.9 & 1.5 & 11.4 & 11.7 \\
$3-6$ & 3 & 25.2 & 1.3 & & 18.5 & 1.5 & 18.4 & 19.1 \\
\hline \hline
\end{tabular}

${ }^{\mathrm{a}}$ For nuclei numbering see scheme 1. All SSCCs are expressed in $\mathrm{Hz}$, and were calculated using CP-B3LYP with the $(11 s, 7 p, 2 d / 5 s, 1 p)[7 s, 6 p, 2 d / 4 s, 2 p]$ basis. In each pair of nuclei the perturbed nucleus is given first. Experimental values from Ref. 35.

$1 s(\mathrm{C} 2)$ orbital, however not the $\sigma(\mathrm{C} 1 \mathrm{H} 3), \sigma(\mathrm{C} 1 \mathrm{H} 4)$, or $1 s(\mathrm{C} 1)$ orbitals (see Table $\mathrm{V}$, small values unequal zero arise from second order effects). The magnitude of the orbital contributions depends on the position of the $\pi$ orbital in the coupling path. It decreases in the following order: (a) $p \pi$ orbitals at both coupling nuclei; (b) at one of the coupling nuclei; (c) at the nucleus (nuclei) next to the coupling nuclei. The corresponding absolute $\pi$ contributions are 5.8 $\left({ }^{*} \mathrm{C}=\mathrm{C}^{*}\right)$ and $2.7\left({ }^{*} \mathrm{CH}\right)$ for ${ }^{1} \mathrm{FC}(\mathrm{CC}), 0.8\left({ }^{*} \mathrm{CC}\right), 0.6$ $\left({ }^{*} \mathrm{CH}^{*}\right)$, or $0.4\left({ }^{*} \mathrm{CH}\right)$ for ${ }^{1} \mathrm{FC}(\mathrm{CH})$ and ${ }^{3} \mathrm{FC}(\mathrm{CCH}), 0.1$ SI units $\left({ }^{*} \mathrm{HC}\right)$ for proton-proton couplings. Here, the starred nuclei denote perturbing and/or responding nucleus. "SI units" means henceforth reduced SSCCs in units of $10^{19} \mathrm{~J} \mathrm{~T}^{-2}$, which are used to show the electronic effect (for $\mathrm{Hz}$ values, see Table $\mathrm{V}$ ). If the induced $\pi$ contribution is associated with a valence orbital, the sign of the contribution can be determined with a Dirac vector model (one-bond coupling, +; two-bond coupling, -; etc.). For $\pi$ contributions induced by the core orbital the opposite sign applies.

The analysis of the $\operatorname{FC}(\pi)$ terms clearly reveals that the spin polarization via the $\pi$ system follows a simple pattern. For example, the total values of the $\mathrm{FC}$ terms of ${ }^{1} \mathrm{~J}(\mathrm{C} 1 \mathrm{H} 3)$ $(153.4 \mathrm{~Hz})$ and ${ }^{2} \mathrm{~J}(\mathrm{C} 2 \mathrm{C} 1 \mathrm{H} 3)(0.6 \mathrm{~Hz}$, Table IV) differ by more than a factor of 100 . The corresponding $\mathrm{FC}(\pi)$ terms, in contrast, differ by less than $20 \%$ in absolute magnitude ( 2.9 and $-2.4 \mathrm{~Hz}$; Table IV) because similar types of contributions are involved: $\mid\left[\left({ }^{*} \mathrm{CC}\right)+\left({ }^{*} \mathrm{CH}^{*}\right)+\left({ }^{*} \mathrm{CH}\right)-1 s(\mathrm{C})\right]$ $-\left[\left({ }^{*} \mathrm{CC}\right)+\left({ }^{*} \mathrm{CH}\right)-1 s(\mathrm{C})\right]|=|\left({ }^{*} \mathrm{CH}^{*}\right)-(* \mathrm{CH}) \mid$

$\approx|0.2| \mathrm{Hz}$. For the geminal and vicinal $\mathrm{H}-\mathrm{H}$ coupling constants, this model is even more strongly supported: All three $\mathrm{H}-\mathrm{H}$ coupling constants are $1.5 \mathrm{~Hz}$ in absolute values, with signs following the Dirac vector model.

\section{B. Comparison of passive $\pi$ contributions obtained with different methods: Basis set dependence}

Although the SSCCs calculated with the CP-DFT method agree reasonably with measured values, this does not provide any proof that the calculated $\pi$ contributions of the various SSCCs are reasonable. In Table VI, the values obtained in this work are compared with INDO/SOS2 $\pi$ contributions calculated by Fukui and co-workers. ${ }^{35,36}$ The results of Contreras and co-workers ${ }^{33,34}$ are comparable to those of Fukui and therefore, we will consider here just the INDO/SOS $2 \pi$ contributions.
The comparison reveals that the $\pi$ contributions to the FC term of $\mathrm{H}-\mathrm{H}$ coupling constants agree well for the semiempirical INDO/SOS2 and the CP-DFT/B3LYP method, even though the values for the total FC terms differ strongly between the two methods. In contrast, for $\mathrm{C}-\mathrm{H}$ and $\mathrm{C}-\mathrm{C}$ SSCCs INDO/SOS2 tends to overestimate the $\mathrm{FC}(\pi)$ contribution by a factor of $\approx 1.5$ (C-H SSCCs) or $2.5-4$ (C-C SSCCs). As seen from Table $\mathrm{V}$, the $\pi$-coupling mechanism between protons does not involve any $1 s(\mathrm{C})$ orbital, in distinction to the case of SSCCs $\mathrm{C}-\mathrm{H}$ and $\mathrm{C}-\mathrm{C}$. The spin polarization of the $1 s(\mathrm{C})$ orbitals, however, requires an appropriate level of theory and an extended basis set for a proper description. This leads to the conjecture that the observed deviations for the $\mathrm{FC}(\pi)$ terms $\mathrm{C}-\mathrm{C}$ and $\mathrm{C}-\mathrm{H}$ are connected to the impact of the $1 \mathrm{~s}(\mathrm{C})$ orbitals. We recalculated therefore the SSCCs of ethylene with Pople's 3-21G basis set. ${ }^{49}$ The results confirm the trends observed for the $\mathrm{FC}(\pi)$ contributions from Fukui's INDO/SOS2 calculations. ${ }^{35,36}$ In Table VII, the passive $\pi$ part of each orbital contribution to ${ }^{1} \mathrm{FC}(\mathrm{C} 1 \mathrm{C} 2)$ of ethylene are compared for the $[7 s, 6 p, 2 d / 4 s, 2 p]$ basis set used in this work (large basis set, A) and the valence DZ basis set 3-21G (small basis set, B). Orbital contributions are given as defined in Eq. (22).

Table VII reveals that the $1 s(\mathrm{C} 2) \mathrm{FC}(\pi)$ contribution is strongly basis-set dependent, amounting to $-3.9 \mathrm{~Hz}$ for basis set $\mathrm{A}$ as compared to $-1.3 \mathrm{~Hz}$ for basis set $\mathrm{B}$. The $\sigma(\mathrm{C} 2 \mathrm{H})$ contribution is by $1.4 \mathrm{~Hz}$ and the $\sigma(\mathrm{C} 1 \mathrm{C} 2)$ contribution by $1.0 \mathrm{~Hz}$ larger for basis B than for basis A.

Figures 4(a) (large basis A), 4(b) (small basis B), and

TABLE VII. The impact of the basis set on the passive $\pi$ contributions to the $\mathrm{FC}$ term of ${ }^{1} \mathrm{~J}(\mathrm{C} 1 \mathrm{C} 2)$ in ethylene. ${ }^{a}$

\begin{tabular}{lrrrrrr}
\hline \hline & \multicolumn{3}{c}{$\mathrm{FC}$} & & \multicolumn{2}{c}{$\mathrm{FC}(\pi)$} \\
\cline { 2 - 3 } Orbital & \multicolumn{1}{c}{$\mathrm{A}$} & \multicolumn{1}{c}{$\mathrm{B}$} & & \multicolumn{1}{c}{$\mathrm{A}$} & \multicolumn{1}{c}{$\mathrm{B}$} & \multicolumn{1}{c}{$\Delta$} \\
\hline $1 s(\mathrm{C} 2)$ & -7.69 & -2.14 & & -3.88 & -1.27 & -2.60 \\
$1 s(\mathrm{C} 1)$ & -0.10 & 0.04 & & 0.00 & 0.00 & 0.00 \\
$\sigma(\mathrm{C} 2 \mathrm{H})$ & -19.51 & -13.21 & & 2.02 & 3.36 & -1.35 \\
$\sigma(\mathrm{C} 1 \mathrm{C} 2)$ & 146.34 & 107.32 & & 4.39 & 5.39 & -1.01 \\
$\sigma(\mathrm{C} 1 \mathrm{H})$ & -10.24 & -7.48 & & -0.03 & -0.04 & 0.02 \\
Total & 79.06 & 63.86 & & 4.49 & 10.75 & -6.26 \\
\hline
\end{tabular}

${ }^{a}$ All values in Hz. $\Delta$ denotes the difference $\mathrm{FC}(\pi) / \mathrm{A}-\mathrm{FC}(\pi) / \mathrm{B}$. FC terms calculated at the CP-B3LYP level of theory with the $[7 s, 6 p, 2 d / 4 s, 2 p]$ basis set (basis A) and the 3-21G basis set (basis B). 


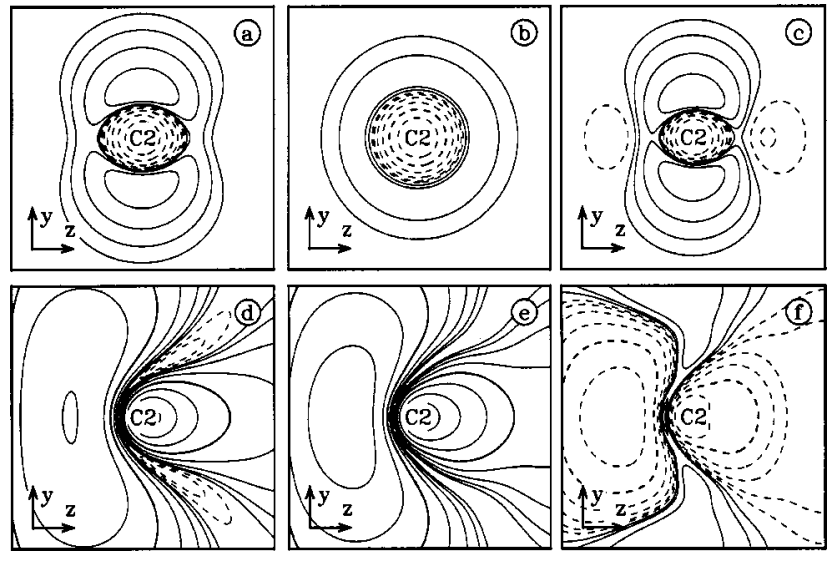

FIG. 4. The FC spin polarization of the $\pi(\mathrm{C} 1 \mathrm{C} 2)$ orbital and its impact on the FC spin polarization of the $1 s(\mathrm{C} 2)$ orbital. The plots were done in the plane through the $\mathrm{CC}$ bond perpendicular to the molecular plane for an area of $1.2 \AA$ by $1.2 \AA$ around $\mathrm{C} 2$. The $z$ axis is along the $\mathrm{CC}$ bond. For the choice of the contour lines, see caption to Fig. 2. The difference of the spin densities are scaled by a factor of 100 . (a) $\mathrm{FC}(\pi)$ spin polarization of $1 s(\mathrm{C} 2)$. B3 $\mathrm{LYP} /[7 s, 6 p, 2 d / 4 s, 2 p]$ calculation. (b) $\mathrm{FC}(\pi)$ spin polarization of $1 s(\mathrm{C} 2)$. B3LYP/3-21G calculation. (c) Difference of the spin densities shown in (a) and (b). (d) $\mathrm{FC}(\pi)$ spin polarization of $\sigma(\mathrm{C} 1 \mathrm{C} 2)$. $\mathrm{B} 3 \mathrm{LYP} /[7 s, 6 p, 2 d / 4 s, 2 p]$ calculation. (e) $\mathrm{FC}(\pi)$ spin polarization of $\sigma(\mathrm{C} 1 \mathrm{C} 2)$. B3LYP/3-21G calculation. (f) Difference of the spin densities shown in (d) and (e).

4(c) (difference A-B) show the impact of the passive $\pi(\mathrm{C} 1 \mathrm{C} 2)$ contribution on the $1 s(\mathrm{C} 2)$ orbital in a region of $1.2 \AA \times 1.2 \AA$ around $\mathrm{C} 2$. As discussed in connection with Figs. 2 and 3, the spin polarization of $\pi(\mathrm{C} 1 \mathrm{C} 2)$ leads to an $\alpha$ surplus spin density in the $\pi$ space around $\mathrm{C} 2$ [Figs. 2(b) and 3(b)]. For basis set A [Fig. 4(a)], the $1 s(\mathrm{C} 2)$ orbital obtains more $\alpha$ spin density in that part of the core region where the $\pi(\mathrm{C} 1 \mathrm{C} 2)$ orbital has still a sizable amplitude. This is in line with the intra-atomic Hund rule according to which the two orbitals maximize their mutual exchange energy. Due to Pauli pairing of the $1 s$ electrons, the $\alpha$ surplus in the outer core region is compensated by a $\beta$ surplus in the inner core region [where the $\pi(\mathrm{C} 1 \mathrm{C} 2)$ orbital is not present]. This gives rise to a large negative spin density of $1 s(\mathrm{C} 2)$ at $\mathrm{C} 2$ and accounts for the large negative contribution of $1 s(\mathrm{C} 2)$ to $\operatorname{FC}(\pi)$ [see Fig. 3(b)].

The small basis set B [Fig. 4(b)] is not able to describe the spin polarization of the $1 s(\mathrm{C} 2)$ orbital properly. One obtains an $\alpha$ surplus density in the outer valence region and a spherical $\beta$ surplus density in a region with a radius of about $0.25 \AA$ around $\mathrm{C} 2$, which no longer reflects Pauli pairing of the core electrons. As this $\beta$ surplus density is less concentrated than for the large basis $\mathrm{A}$, its negative contribution to $\mathrm{FC}(\pi)$ is absolutely smaller (Table VII; note that for the FC mechanism only the spin density at the contact surface of the nucleus counts). Actually, the extra spin density and thus the extra attractive potential induced by the $\pi(\mathrm{C} 1 \mathrm{C} 2)$ orbital leads to an anisotropic component of the FC spin density, which has $d_{y^{2}}$ character [see Fig. 4(a)]. A proper description of the spin polarization requires virtual $d$ functions, which are not available in the small basis B [Fig. 4(b)]. The increased flexibility of the large basis A as compared to basis B [see difference of the FC spin density shown in Fig. 4(c)] results in a $\beta$ surplus in a small region around $\mathrm{C} 2$, surrounded by a region with $\alpha$ spin surplus.

The basis set dependence of the $\operatorname{FC}(\pi)$ contributions from the $\sigma(\mathrm{C} 1 \mathrm{C} 2)$ [Figs. 4(d), 4(e), and 4(f) $], \sigma(\mathrm{C} 2 \mathrm{H} 5)$ and $\sigma(\mathrm{C} 2 \mathrm{H} 6)$ orbitals can be rationalized similarly as that for the $1 s(\mathrm{C} 2)$ orbital. However, there is a difference with regard to the $1 s(\mathrm{C} 2)$ contribution as the spin polarization around $\mathrm{C} 2$ results from two different mechanisms. (1) Intra-atomic mechanism: The spin polarization of the $\pi(\mathrm{C} 1 \mathrm{C} 2)$ density implies an increase of $\alpha$ spin density in that part of the $\sigma(\mathrm{C} 1 \mathrm{C} 2)$ and $\sigma(\mathrm{C} 2 \mathrm{H})$ orbitals surrounding $\mathrm{C} 2$ and overlapping with the $\pi$ region [Fig. 3(a), mechanism 1]. (2) Interatomic mechanism: There is also an increase in $\alpha$ spin density at $\mathrm{C} 2$ due to Pauli coupling in the $\sigma(\mathrm{C} 1 \mathrm{C} 2)$ orbital [Fig. 3(a), mechanism 2], which influences Pauli pairing in the $\pi$ orbital by increasing it. [Note that the direct $\sigma(\mathrm{C} 1 \mathrm{C} 2)$ effect, is excluded from Figs. 4(d) and 4(e) because these are difference plots generated by subtracting the $\sigma(\mathrm{CC}) \mathrm{FC}$ spin density for the frozen $\pi$ orbital from the normal $\sigma(\mathrm{CC}) \mathrm{FC}$ spin density.] The interatomic mechanism can be reasonably described by the small basis because it takes place in the bond region, for which any small basis such as B is optimized.

The intra-atomic mechanism implies that the region close to nucleus $\mathrm{C} 2$ is correctly described. The large basis A fills out the valence space and allows an even distribution of the FC spin density into outer parts of this region [Fig. 4(d)] whereas the small basis set $\mathrm{B}$ gives a rigid, compact spin density distribution in the inner valence region around $\mathrm{C} 2$ [Fig. 4(e)]. This is confirmed by the difference of the spin density distribution A-B [Fig. 4(f)], which is predominantly negative around $\mathrm{C} 2$ in line with the larger $\alpha$ spin density obtained with basis B.

Similar diagrams are obtained for the $\mathrm{FC}(\pi)$ contributions from the $\sigma(\mathrm{C} 2 \mathrm{H} 5)$ and $\sigma(\mathrm{C} 2 \mathrm{H} 6)$ orbitals. This means that the positive passive $\pi$ contributions of the three $\sigma$ orbitals are exaggerated by basis B by $3.7 \mathrm{~Hz}$ (Table VII). Together with the underestimation of the negative $1 s(\mathrm{C} 2)$ contribution, basis B exaggerates the $\pi$ part of the ${ }^{1} \mathrm{FC}(\mathrm{CC})$ by $6.3 \mathrm{~Hz}$ (Table VII). Hence, INDO/SOS2 calculations, which are based on a minimal basis set description must fail in the same way as basis B as soon as a detailed description around the core and inner valence region of perturbing and responding nucleus is required: Too large $\pi$ contributions are predicted (Table VI).

The situation is different for proton-proton coupling. The details of the spin polarization close to the $\mathrm{C}$ nuclei do not play any major role for the transport of the spin through the $\pi$ system, e.g., the $1 s(\mathrm{C})$ contributions to the $\pi$ mechanism are zero (Table V). The $\mathrm{FC}(\pi)$ mechanism can be rationalized with simple Dirac vector models. In some sense, this part of the mechanism is "coarse grained" and can thus be described reasonably by less sophisticated methods such as INDO ${ }^{33-36}$ or DFT with a small basis set. We conclude that the passive $\pi$-contribution calculated with basis A should be reasonable whereas larger basis sets with more functions in the core regions should lead to further (small) improvements. Also, we note that the analysis of the basis set dependence provides indirect proof for the reliability of the 
$\mathrm{FC}(\pi)$ spin-spin coupling mechanism developed in this work.

\section{CONCLUSIONS}

In this work, we have developed a theory for a detailed decomposition of each of the four Ramsey terms of the isotropic indirect SSCC where the essentials of this theory are summarized in points (a) $-(\mathrm{g})$ :

(a) The theory can be expressed in terms of any kind of orbitals, however for practical reasons we have chosen LMOs, which lead to bond, lone pair, core, etc., contributions familiar to the chemist.

(b) The theory as presented in this work is based on the formulation of first order orbitals and the CP-DFT equations in form of LMOs [Eqs. (3) and (5)] and the decomposition of the first order Fock operator into a sum of orbital Fock operators [Eq. (4)]. The relationship between the CP-DFT coefficients $C_{a k}^{(B)}$ derived in Eq. (5) and the orbital Hessian is established [see Eq. (6)] so that a comparison with previous methods just based on the orbital Hessian can be made.

(c) A simple form of the new theory for analyzing SSCCs, called J-OC-PSP1, has been formulated in terms of one- and two-orbital contributions based on active orbitals [Eqs. (15), (21), (22), and Appendix B]. The oneorbital terms account for Ramsey distortion of the orbital density, which can imply repolarization and delocalization effects, as well as self-exchange interactions. The two-orbital contributions comprise steric exchange interactions and resonance interactions between the orbitals.

(d) Coupling paths have been described by orbital paths, which is one of the advantages of using LMOs. An orbital path is a chain of orbitals leading from the perturbing nucleus $\mathrm{B}$ to the responding nucleus $\mathrm{A}$. One can distinguish between active and passive orbitals according to their position in a specific orbital path. Active orbitals are directly perturbed by nucleus B and/or interact directly with the responding nucleus A whereas passive orbitals interact only with other orbitals (active or passive), but not with the coupling nuclei.

(e) Passive orbitals contribute to the spin-spin coupling mechanism via three-, four-, etc., $n$-orbital paths, and their orbital contributions can be calculated with J-OC-PSP2 as shown in Eqs. (25) and (26). For the purpose of determining passive orbital contributions, the concept of frozen orbitals has been introduced, i.e., orbitals that are no longer included into the CP-DFT equations. Hence, if a particular orbital is frozen, both its active and passive contributions will be eliminated from the SSCC. Formulas are given to determine active and passive part of three-orbital contributions. A generalization to multiorbital contributions is straightforward.

(f) The passive orbitals are involved in echo effects and transport effects, which can be identified by J-OCPSP2 analysis. The former imply cyclic, the latter acyclic orbital paths. (g) The concept of active and passive orbitals can be refined in the way that only excitations from an occupied orbital into selected virtual orbitals are set passive. In this case, $P_{a k, a^{\prime} k^{\prime}}^{\text {pass }}$ of Eq. (20) projects out individual excitations $k \rightarrow a$ instead of all excitations from a given orbital $k$. Depending on the nature of the virtual orbital $a$, special spin-polarization effects can be distinguished (repolarization or delocalization of the spin density of a bond orbital; etc. ${ }^{20}$ ). In the present paper, no use of this possibility was made.

Conclusions (a) - (g) apply to both the FC, PSO, and SD terms, however the role of passive orbitals, echo, and transport effects is best seen for the FC part. Application of the J-OC-PSP2 method to the SSCCs in ethylenes reveals that NMR spin-spin coupling is based on a rather complex mechanism, even if just FC spin-spin coupling dominates. Both active and passive orbital contributions to the FC term have to be considered, which can offer the FC spin polarization a multitude of paths in the molecule. In this work, we have concentrated on the $\pi$ mechanism of FC spin-spin coupling, which is based on the passive contributions of the $\pi$ orbitals. Its investigation has led to the description of a number of interesting effects.

(1) The FC coupling mechanism is predominantly based on exchange interactions (as contained in $\tilde{F}$ ). Correlation effects (also contained in $\tilde{F}$ ) play a minor role. Coulomb interactions do not play any role because spinpolarization (expressed by the first order density) does not change the total electron density. Thus, even though FC coupling is mediated by the spin density and thus is a one-particle property, the exchange interaction as a two-electron process is crucial for its description. This implies also that a balanced description of the exchange interactions is important for an accurate calculation of the FC term, which in turn is one of the reasons for the good performance of hybrid exchange functionals in this respect. $^{16}$

(2) There are three basic exchange effects that explain the FC spin coupling mechanism across a double bond (or other bonds): (a) Fermi coupling between nuclear spin moment and electron spin moment involves just $1 s$ and $2 s$ electrons. (b) Intra-atomic exchange coupling (to optimize exchange interactions; intra-atomic Hund rule) channels $s$-spin polarization into all bonds and from the $\sigma$ into the $\pi$ space. (c) Pauli coupling of the two electrons occupying a $\sigma$ - or $\pi$-bonding orbital requires that one electron possesses $\alpha$ the other $\beta$ spin. Hence, if one spin is preferred at one of the coupling nuclei because of (b), the other spin will be preferentially found at the other nucleus. This is an interatomic transport mechanism and leads to the transmission of spin information from one nucleus to the other. Despite the important role of Pauli pairing in this connection, we prefer to consider the interatomic spin-transport effect also as an exchange effect, which results from the withdrawal of a preferred spin from the second atom to optimize exchange interactions at the first atom. 
(3) The three basic spin transfer mechanisms lead to the simplest coupling mechanism and allow a distinction between active and passive orbital contributions: For an active contribution the orbital in question must lead to significant FC spin densities at the perturbing or the responding nucleus (or at both). A passive contribution results when an orbital transmits spin polarization from one (partially) active to another (partially) active orbital. This can be at the stage of the intra-atomic exchange interactions or at the stage of interatomic exchange interactions, e.g., between the $\sigma$ and the $\pi$ orbital of a CC double bond.

(4) The $\pi$ orbitals make a passive contribution to the FC coupling mechanism of the SSCCs. This contribution can arise in two ways: Either a $\pi$ orbital gets spin polarized by an active orbital and causes in turn a change of that active orbital ("echo mechanism"), or an active orbital carries spin information to a $\pi$ orbital, which forwards it to another spin orbital ("transport mechanism"). The echo mechanism requires only one active orbital, hence its impact can be indirectly seen in the Ramsey distortion, and it is contained in the one-orbital terms at the J-OC-PSP1 level of theory. The transport mechanism, in contrast, connects two different active orbitals and will thus be subsumed in the two-orbital terms (J-OC-PSP1) whereas it appears explicitly in the threeorbital terms in J-OC-PSP2. Both mechanisms can play an important role for the FC coupling. By combining a number of orbital-selected SSCC calculations for the molecule under investigation, the passive contribution of a single $\pi$ orbital or the $\pi$ system as a whole can be determined quantitatively.

The passive $\pi$ orbitals play an important role for the long-range SSCCs in polyenes as we will investigate in a separate paper. The so-called $\pi$ mechanism for FC spin-spin coupling in unsaturated hydrocarbons is carried by the spin polarization of the $\pi$ orbitals. Passive $\sigma$ orbital contributions can contribute to the PSO and SD spin-spin coupling mechanism. These effects will also be investigated in a separate paper.

\section{ACKNOWLEDGMENTS}

Calculations were done on the supercomputers of the Nationellt Superdatorcentrum (NSC), Linköping, Sweden. D.C. thanks the NSC for a generous allotment of computer time. J.G. thanks Carl Tryggers Stiftelse for financial support.

\section{APPENDIX A: DERIVATION OF EQ. (3)}

The CP-DFT equations given in Eq. (3) are derived by starting from the general form of the KS equations

$$
\begin{aligned}
& (1-\hat{P}) \hat{F}\left|\psi_{k}\right\rangle=0, \\
& \hat{P}=\sum_{l}^{\text {occ }}\left|\psi_{l}\right\rangle\left\langle\psi_{l}\right| .
\end{aligned}
$$

The representation (A1) has the advantages that it is valid for any type of the orbital (canonical MOs, LMOs, etc.) and that it does not contain the Lagrange multipliers $\left(\epsilon_{k}\right.$ or $F_{k l}$ ) explicitly. Expanding Eqs. (A1) and (A2) with respect to a perturbation $(\mathrm{B})$ and collecting all first-order terms gives

$$
\begin{gathered}
-\hat{P}^{(B)} \hat{F}^{(0)}\left|\psi_{k}^{(0)}\right\rangle+\left(1-\hat{P}^{(0)}\right) \hat{F}^{(B)}\left|\psi_{k}^{(0)}\right\rangle \\
+\left(1-\hat{P}^{(0)}\right) \hat{F}^{(0)}\left|\psi_{k}^{(B)}\right\rangle=0 .
\end{gathered}
$$

Here,

$$
\begin{aligned}
& \hat{P}^{(0)}=\sum_{l}^{\text {occ }}\left|\psi_{l}^{(0)}\right\rangle\left\langle\psi_{l}^{(0)}\right|, \\
& \hat{P}^{(B)}=\sum_{l}^{\text {occ }}\left|\psi_{l}^{(B)}\right\rangle\left\langle\psi_{l}^{(0)}\left|+\sum_{l}^{\text {occ }}\right| \psi_{l}^{(0)}\right\rangle\left\langle\psi_{l}^{(B)}\right| .
\end{aligned}
$$

Multiplying Eq. (A3) from the left with the canonical virtual $\mathrm{MO}\left\langle\varphi_{a}^{(0)}\right|$ yields

$$
\begin{aligned}
& -\sum_{l}\left\langle\varphi_{a}^{(0)} \mid \psi_{l}^{(B)}\right\rangle \underbrace{\left\langle\psi_{l}^{(0)}\left|\hat{F}^{(0)}\right| \psi_{k}^{(0)}\right\rangle}_{=F_{k l}^{(0)}}-\sum_{l} \underbrace{\left\langle\varphi_{a}^{(0)} \mid \psi_{l}^{(0)}\right\rangle}_{=0} \\
& \quad \times\left\langle\psi_{l}^{(B)}\left|\hat{F}^{(0)}\right| \psi_{k}^{(0)}\right\rangle+\left\langle\varphi_{a}^{(0)}\left|\hat{F}^{(B)}\right| \psi_{k}^{(0)}\right\rangle \\
& +\underbrace{\left\langle\varphi_{a}^{(0)}\left|\hat{F}^{(0)}\right|\right.}_{=\varepsilon_{a}\left\langle\psi_{a}^{(0)}\right|} \psi_{k}^{(B)}\rangle=0 \\
& \epsilon_{a}\left\langle\varphi_{a}^{(0)} \mid \psi_{k}^{(B)}\right\rangle-\sum_{l} F_{k l}^{(0)}\left\langle\varphi_{a}^{(0)} \mid \psi_{l}^{(B)}\right\rangle+\left\langle\varphi_{a}^{(0)}\left|\hat{F}^{(B)}\right| \psi_{k}^{(0)}\right\rangle=0
\end{aligned}
$$

which can be rearranged into

$$
\begin{aligned}
-\epsilon_{a} & \left\langle\varphi_{a}^{(0)} \mid \psi_{k}^{(B)}\right\rangle+F_{k k}^{(0)}\left\langle\varphi_{a}^{(0)} \mid \psi_{k}^{(B)}\right\rangle \\
& =\left\langle\varphi_{a}^{(0)}\left|\hat{F}^{(B)}\right| \psi_{k}^{(0)}\right\rangle-\sum_{l, l \neq k} F_{k l}^{(0)}\left\langle\varphi_{a}^{(0)} \mid \psi_{l}^{(B)}\right\rangle .
\end{aligned}
$$

Dividing Eq. (A6) by $\left(F_{k k}^{(0)}-\epsilon_{a}\right)$ leads to Eq. (A7),

$\left\langle\varphi_{a}^{(0)} \mid \psi_{k}^{(B)}\right\rangle=\frac{\left\langle\psi_{a}^{(0)}\left|F^{(B)}\right| \psi_{k}^{(0)}\right\rangle-\Sigma_{l, l \neq k} F_{k l}^{(0)}\left\langle\psi_{a}^{(0)} \mid \psi_{l}^{(B)}\right\rangle}{F_{k k}^{(0)}-\epsilon_{a}}$

which by multiplication with $\left|\phi_{a}\right\rangle$ and summation over $a$ yields Eq. (3).

\section{APPENDIX B: CALCULATION OF THE $K_{\mathrm{AB}}^{\mathrm{PSP} 1}(k \leftrightarrow l)$}

In this Appendix it is shown how the two-orbital contributions $K_{\mathrm{AB}}^{\mathrm{PS} 1}(k \leftrightarrow l)$ can be calculated efficiently.

In a CP-DFT calculation, coefficients $C_{a k}^{(B)}$ depend linearly on elements $h_{a k}^{(B)}$. Because of

$$
h_{a k}^{(N), \text { pass }}[\neg(k, l)]=h_{a k}^{(N), \text { pass }}[\neg k]+h_{a k}^{(N), \text { pass }}[\neg l]
$$

$(N=A, B)$ it is

$$
C_{a k}^{(B), \operatorname{pass}}[\neg(k, l)]=C_{a k}^{(B), \operatorname{pass}}[\neg k]+C_{a k}^{(B), \operatorname{pass}}[\neg l] .
$$

The SSCC $K_{\mathrm{AB}}^{\mathrm{pass}}[\neg(k, l)]$ becomes therefore 


$$
\begin{aligned}
K_{\mathrm{AB}}^{\mathrm{pass}}[\neg(k, l)] & \\
= & \sum_{a k^{\prime}} C_{a k^{\prime}}^{(B), \text { pass }}[\neg(k, l)] h_{a k^{\prime}}^{(A), \text { pass }}[\neg(k, l)] \\
= & \sum_{a} C_{a k}^{(B), \text { pass }}[\neg(k, l)] h_{a k}^{(A)}+\sum_{a} C_{a l}^{(B), \text { pass }}[\neg(k, l)] h_{a l}^{(A)} \\
= & \sum_{a} C_{a k}^{(B), \text { pass }}[\neg k] h_{a k}^{(A)}+\sum_{a} C_{a k}^{(B), \text { pass }}[\neg l] h_{a k}^{(A)} \\
& +\sum_{a} C_{a l}^{(B), \text { pass }}[\neg k] h_{a l}^{(A)}+\sum_{a} C_{a l}^{(B), \text { pass }}[\neg l] h_{a l}^{(A)} .
\end{aligned}
$$

Considering that

$$
K_{\mathrm{AB}}^{\mathrm{pass}}[\neg q]=\sum_{a} C_{a q}^{(B), \operatorname{pass}}[\neg q] h_{a q}^{(A)},
$$

$(q=k, l)$ Eq. (B3) can be rewritten as

$K_{\mathrm{AB}}^{\mathrm{pass}}[\neg(k, l)]$

$$
\begin{aligned}
= & K_{\mathrm{AB}}^{\mathrm{pass}}[\neg p]+K_{\mathrm{AB}}^{\mathrm{pass}}[\neg q]+\sum_{a} C_{a k}^{(B), \operatorname{pass}}[\neg l] h_{a k}^{(A)} \\
& +\sum_{a} C_{a l}^{(B), \operatorname{pass}}[\neg k] h_{a l}^{(A)},
\end{aligned}
$$

which together with Eq. (22) gives

$$
\begin{aligned}
K_{\mathrm{AB}}^{\mathrm{PSP} 1}(k \leftrightarrow l)= & \sum_{a} C_{a l}^{(B), \text { pass }}[\neg k] h_{a l}^{(A)} \\
& +\sum_{a} C_{a k}^{(B), \text { pass }}[\neg l] h_{a k}^{(A)} .
\end{aligned}
$$

Thus, $K_{\mathrm{AB}}^{\mathrm{PSP} 1}(k \leftrightarrow l)$ can be derived as a by-product of the calculation of $K_{\mathrm{AB}}^{\mathrm{PSP} 1}(k)$ and $K_{\mathrm{AB}}^{\mathrm{PSP} 1}(l)$. Evidently, the first term in Eq. (B6) corresponds to the path in Eq. (18), the second one to that in Eq. (18).

If orbitals are frozen there will be no longer a linear relationship between $h_{a k}^{(B)}$ and $C_{a k}^{(B)}$. Hence, there is no analogue to Eq. (B6) for $K_{\mathrm{AB}}^{\mathrm{PSP} 2}(k \leftrightarrow l)$.

${ }^{1}$ See, e.g., Encyclopedia of Nuclear Magnetic Resonance, edited by D. M. Grant and R. K. Harris (Wiley, Chichester, 1996).

${ }^{2}$ J. A. Pople, W. G. Schneider, and H. J. Bernstein, High-Resolution Nuclear Magnetic Resonance (McGraw-Hill, New York, 1959).

${ }^{3}$ J. W. Emsley, J. Feeney, and L. H. Sutcliffe, High Resolution Nuclear Magnetic Resonance Spectroscopy (Pergamon, Oxford, 1966).

${ }^{4}$ H. Günther, NMR-Spectroscopy (Thieme, New York, 1983).

${ }^{5} \mathrm{H}$. O. Kalinowski, S. Berger, and S. Braun, ${ }^{13} \mathrm{C}$-NMR-Spektroskopie (Georg Thieme, Verlag Stuttgart, 1984).

${ }^{6}$ L. M. Jackman and S. Sternhell, International Series of Monographs in Organic Chemistry, Vol. 5, Applications of Nuclear Magnetic Resonance Spectroscopy in Organic Chemistry (Pergamon, Oxford, 1969).

${ }^{7}$ R. H. Contreras and J. C. Facelli, Annu. Rep. NMR Spectrosc. 27, 255 (1993).
${ }^{8}$ R. H. Contreras and J. E. Peralta, Prog. Nucl. Magn. Reson. Spectrosc. 37, 312 (2000); R. H. Contreras, J. E. Peralta, C. G. Biribet, M. C. Ruiz De Azua, and J. C. Facelli, Annu. Rep. NMR Spectrosc. 41, 55 (2000).

${ }^{9}$ M. Karplus and D. H. Anderson, J. Chem. Phys. 30, 6 (1959); M. Karplus, ibid. 30, 11 (1959); (c) J. Am. Chem. Soc. 85, 2870 (1963).

${ }^{10}$ N. Muller and D. E. Pritchard, J. Chem. Phys. 31, 768 (1959).

${ }^{11}$ M. D. Newton, J. M. Schulman, and M. M. Manus, J. Am. Chem. Soc. 96, 17 (1974).

${ }^{12}$ K. Frei and H. J. Bernstein, J. Chem. Phys. 38, 1216 (1963).

${ }^{13}$ J. Wardeiner, W. Lüttke, R. Bergholz, and R. Machinek, Angew. Chem., Int. Ed. Engl. 21, 872 (1982).

${ }^{14}$ H. Spiesecke and W. G. Schneider, Tetrahedron Lett. 1961, 468 (1961).

${ }^{15}$ S. Fliszar, Charge Distributions and Chemical Effects (Springer, New York, 1983).

${ }^{16}$ V. Sychrovský, J. Gräfenstein, and D. Cremer, J. Chem. Phys. 113, 3530 (2000).

${ }^{17}$ A. Wu, D. Cremer, A. A. Auer, and J. Gauss, J. Phys. Chem. A 106, 657 (2002).

${ }^{18}$ A. Wu and D. Cremer, Int. J. Mol. Sci. 4, 159 (2003).

${ }^{19} \mathrm{~A}$. Wu and D. Cremer, J. Phys. Chem. A 107, 1797 (2003).

${ }^{20}$ A. Wu, J. Gräfenstein, and D. Cremer, J. Phys. Chem. 107, 7043 (2003).

${ }^{21}$ J. Gräfenstein and D. Cremer, Chem. Phys. Lett. 383, 332 (2004).

${ }^{22}$ J. Gräfenstein and D. Cremer, Chem. Phys. Lett. 387, 415 (2004).

${ }^{23}$ D. Cremer, E. Kraka, A. Wu, and W. Lüttke, ChemPhysChem 5, 349 (2004).

${ }^{24}$ J. Gräfenstein and D. Cremer J. Phys. Chem. A (in press).

${ }^{25}$ J. Gräfenstein, T. Tuttle, and D. Cremer (unpublished).

${ }^{26}$ A. Wu and D. Cremer, Phys. Chem. Chem. Phys. 5, 4541 (2003).

${ }^{27}$ T. Tuttle, E. Kraka, A. Wu, and D. Cremer, J. Am. Chem. Soc. (in press).

${ }^{28}$ T. Tuttle, J. Gräfenstein, A. Wu, E. Kraka, and D. Cremer, J. Phys. Chem. B 108, 1115 (2004)

${ }^{29}$ N. F. Ramsey, Phys. Rev. 91, 303 (1953).

${ }^{30}$ J. N. Murrell, Prog. Nucl. Magn. Reson. Spectrosc. 6, 1 (1971).

${ }^{31}$ M. Barfield, Indirect Coupling: Theory and Applications in Organic Chemistry. Encyclopedia of NMR (Wiley, New York, 1996), p. 2520; M. Barfield and B. Chakrabarty, Chem. Rev. 69, 757 (1969).

${ }^{32}$ J. Kowalewski, Prog. Nucl. Magn. Reson. Spectrosc. 11, 1 (1977); Annu. Rep. NMR Spectrosc. 12, 81 (1982).

${ }^{33}$ A. R. Engelmann, R. H. Contreras, and J. C. Facelli, Theor. Chim. Acta 59, 17 (1981).

${ }^{34}$ A. R. Engelmann, G. E. Scuseria, and R. H. Contreras, J. Magn. Reson. 50, 21 (1982).

${ }^{35}$ H. Fukui, T. Tsuji, and K. Miura, J. Am. Chem. Soc. 103, 3652 (1981).

${ }^{36}$ H. Fukui, K. Miura, K. Ohta, and T. Tsuji, J. Chem. Phys. 76, 5169 (1982).

${ }^{37}$ J. E. Wertz and J. R. Bolton, Electron Spin Resonance, Elementary Theory and Practical Applications (McGraw-Hill, New York, 1972).

${ }^{38}$ R. Bauernschmitt and R. Ahlrichs, J. Chem. Phys. 104, 9047 (1996).

${ }^{39}$ J. E. Peralta, R. H. Contreras, and J. P. Snyder, Chem. Commun. (Cambridge) 2000, 2025 (2000).

${ }^{40}$ S. J. Wilkens, W. M. Westler, J. L. Markley, and F. Weinhold, J. Am. Chem. Soc. 123, 12026 (2001).

${ }^{41}$ A. D. Becke, Phys. Rev. A 38, 3098 (1988).

${ }^{42}$ C. Lee, W. Yang, and R. P. Parr, Phys. Rev. B 37, 785 (1988)

${ }^{43}$ A. D. Becke, J. Chem. Phys. 98, 5648 (1993).

${ }^{44}$ S. Huzinaga, Approximate Atomic Wave Functions (University of Alberta Press, Edmonton AB, Canada, 1971).

${ }^{45}$ W. Kutzelnigg, U. Fleischer, and M. Schindler, NMR-Basic Principles and Progress (Springer, Heidelberg, 1990), Vol. 23, p. 165.

${ }^{46}$ H. C. Allen and E. K. Plyler, J. Am. Chem. Soc. 80, 2673 (1958).

${ }^{47}$ S. F. Boys, Rev. Mod. Phys. 32, 296 (1960).

${ }^{48}$ E. Kraka, J. Gräfenstein, M. Filatov et al., COLOGNE 2003, Göteborg University, Göteborg, 2003.

${ }^{49}$ J. S. Binkley, J. A. Pople, and W. J. Hehre, J. Am. Chem. Soc. 102, 939 (1980). 